\title{
Da Comune a Capitale. Storia dell'identità visiva di Roma
}

\author{
From Municipality to the Capital. History of the visual \\ identity of Rome
}

Paolo Sorrentino

D.Phil em Comunicação da Universidade La Sapienza de Roma, é professor de semiótica na Academia do Traje e da Moda. Sua pesquisa se concentra em discurso, cultura e identidade.) -

Riassunto: La ricerca ricostruisce la storia e i significati dello Stemma del Comune di Roma, dal Medioevo sino ai giorni nostri. L'Ente venne fondato nel 1143 e per tre secoli governò l'Urbe. Di questo periodo, a tutt'oggi, sappiamo pochissimo. La ricerca sulle "identità visive" assume quindi il valore di una testimonianza storica rilevando la precisa "narratività" del Discorso del Comune di Roma: esso si presenta come il vero erede dell'antica Repubblica (il suo Destinante), che lotta per restituire ai Romani (il suo Destinatario) la Libertà, l'Indipendenza e la Ricchezza (gli Oggetti di Valore) sottratti dal Papato (l'Anti-Soggetto). Oggi le strategie di posizionamento del brand cittadino cambiano radicalmente l'assetto del Discorso. Con straordinario ribaltamento, il mito della Cristianità sembra essere più attraente della tradizione della Repubblica. In questo contesto, quale sarà il destino dell'antico Stemma e con esso di Roma?

Parole-chiave: Semiotica, Storia, Roma.

Abstract: Research is reconstructing the history and the signified of the Emblem of the Municipality of Rome, from the middle Ages to the present day. The Municipatility was founded in 1143 and for three centuries governed the Urbe. Very little is known about this period. The research on the "visual identity" used by the Municipality has therefore also the value of a historical testimony revealing the Narrativity of the Discourse of the Municipality: it constitutes the true heir of the Roman Republic (its Addresser) which is fighting to give back to the Romans (its Addressee) the freedom, independence ad wealth (its Objects of Value) withdrawn from the Papacy (anti-Subject). Nowadays, the strategies of placement of the city brand are radically changing the contents of the Discourse. With extraordinary capsizing, the "myth of the Christianity" appears to be more appealing than the "myth of the Republic". In this context, which will be the destiny of the old Emblem and of Rome?

Keywords: Semiotics, History, Rome.s 


\section{Impostazione della ricerca}

\section{Lo Stemma}

Il punto di partenza della ricerca che viene qui presentata, nella quale si prova ad indagare l'identità della città di Roma così come essa viene prodotta attraverso le pratiche di auto-rappresentazione simbolica del suo Comune, è la ricostruzione del valore e del significato dell'antico simbolo col quale la città, a partire dal Medioevo, è stata identificata: il suo storico Stemma, composto da un "corpo", a forma di scudo gotico di colore rosso porpora che reca la sigla in oro annunciante il motto repubblicano Senatus et Populosque Romanus, e da una "testa", sovrastata una corona gigliata che ne incarna il valore di assoluta sovranità.

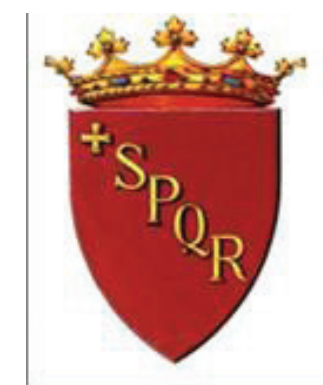

Partire dai "simboli", o più in generale dalle "formazioni semiotiche" (SEDDA, 2012), come ad esempio le "identità visive", per fare una storia di come il Comune ha costruito la sua identità, quella della città, dei cittadini, degli altri attori presenti nel tessuto urbano, e dei rapporti intercorrenti tra questi "termini", è anzitutto un atto di riconoscimento del loro straordinario potere di costruzione, condensazione e rappresentazione dei modi dell'essere e del fare (DE CERTEAU, 1990) di un certo periodo storico, della loro capacità di segnare confini e relative appartenenze (LOTMAN, 1985) territoriali e, a partire da queste funzioni, della loro facoltà di provocare o respingere affetti e passioni, identificazioni e conflitti (GENINASCA, 1997). Lavorare sulle identità della città a partire dai simboli significa dunque indagare la "forma di vita" di cui essi sono "testimoni" in quanto "documenti" e, al tempo stesso, "produttori" in quanto "testi galeotti" capaci cioè di attivare o neutralizzare nei loro "destinatari” sentimenti di amore e di orgoglio, di indifferenza e di freddezza, di odio e di avversione (PEZZINI, 1998).

\section{La periodizzazione}

Il nostro studio su questa storia del Comune di Roma parte dal momento in cui lo Stemma fece la sua comparsa per poi seguirne le vicende nel corso dei processi e degli eventi storici che lo hanno riguardato. Abbiamo così coperto un arco temporale lungo quasi mille anni, dal Medioevo sino ai giorni nostri, giorni nei quali il legame dello "scudo" con la città sembra essere messo in bilico, scavalcato dai nuovi sistemi semiotici di identificazione, e forse superato da nuove - ma incerte - strategie di branding che l'Amministrazione capitolina ha messo in campo nell'ambito delle proprie "semio-politiche" dell'identità (SEDDA, 2012). Tale periodizzazione ha segnato la divisione delle sezioni di questo articolo: nella prima ci dedicheremo al periodo medioevale, con una attenzione maggiormente portata alla sua storia, anche per ridurre la distanza 
che da esso ci separa; mentre nella seconda parte, la maggiore familiarità con la storia moderna e attuale, ci consentirà di concentrarci preminentemente sulle nuove forme di comunicazione istituzionale e sui loro effetti di senso; infine, nelle "conclusioni", il confronto tra i due periodi ci offrirà degli spunti di interesse per l'attualità, mostrandoci le trasformazioni dei processi culturali e delle identità sociali.

Sin da subito possiamo dire che la nostra segmentazione in periodi non ricalca la divisione in grandi "epoche" assegnata alla Storia dalla Storiografia. Infatti, se pure abbiamo usato categorie storiche, la scomposizione in periodi segue la rottura interna ad un discorso che sarebbe ingenuo considerare omogeneo solo perché enunciato dall'identico soggetto. Da un punto di vista semiotico, ciò che è evidentemente mutato, nel corso della sua storia, è il "genere discorsivo" adottato dal Comune. Esso infatti enuncia dapprima un discorso di resistenza, di protesta e di rivoluzione rispetto ad un potere, quello papale, col quale dovrà costantemente commisurare le proprie istanze di governo sulla città, per poi mutare radicalmente il proprio discorso a partire dall'Unità d'Italia.

Il primo periodo analizzato rappresenta quindi un'occasione di studio della narratività e dei linguaggi del discorso di genere rivoluzionario nel quale il soggetto mette in campo una serie di strategie, di tattiche e di tecniche tese alla realizzazione di un programma di sottrazione e di conquista del potere. Rientrano in questo genere di discorso i problemi di legittimazione della propria istanza di governo che si risolvono principalmente in un processo di creazione del consenso. Un processo che trascina il soggetto dallo spazio della sovversione a quello della sua istituzionalizzazione. Vedremo infatti come in tale Discorso il soggetto talloni due vie che si incrociano di continuo ma che è bene tenere distinte almeno in fase di analisi: da una parte esso è preoccupato di darsi una definizione che "segue" il Diritto, e cioè di trovare una propria collocazione all'interno delle Istituzioni riconosciute dalla tradizione giuridica; dall'altra mette in campo delle strategie tese a provocare l'adesione al proprio progetto in una fascia della popolazione che sia la più ampia possibile. Il tutto viene giocato sull'unico filo dei miti e dei linguaggi, i quali traggono la loro forza e il loro significato da uno scontro dialettico che nel nostro caso vede fronteggiarsi il Comune come soggetto nascente e il Papato come soggetto regnante. È interessante notare che entrambi i soggetti del conflitto, al fine di legittimare il proprio potere, fanno appello ognuno ad un diverso mito del passato, con la promessa più o meno esplicita di riportare la popolazione ai fasti e alla vita di quell'epoca: il Comune si autorappresenta come l'erede delle istanze dell'antica Repubblica romana, mentre il Papato come il legittimo successore del primo Impero romano. Di conseguenza, entrambi i soggetti adottano un linguaggio nel quale vengono convocati temi e figure relative al proprio passato di riferimento. Nel secondo periodo, il Comune, ormai legittimato dalla Repubblica italiana, non dovrà più difendere il proprio potere dagli attacchi del papato. Il discorso che prenderemo in analisi sarà quindi di genere "istituzionale", nel quale necessariamente i temi dominanti non saranno più riconducibili a logiche avversative, ma semmai a logiche di mantenimento e 
di gestione del potere. In questo caso la legittimazione del Comune passa per un "buon governo" (FOUCAULT, 1994) della città e del territorio e, insieme ad essi, della loro "immagine". Vedremo quindi come il Comune dovrà "presentificare" se stesso all'interno del territorio, apponendovi le proprie "marche istituzionali", e contemporaneamente dovrà gestire l'immagine sua e della città al fine di renderla "competitiva sul mercato".

\section{Il corpus e la prospettiva}

Come abbiamo accennato, lungo il nostro percorso ci concentreremo sulla costruzione e sulle trasformazioni storiche della soggettività degli attori inscritti nel Discorso del Comune di Roma. Vedremo quindi come l'Enunciatore - usiamo questo termine e non quello di "autore" in quanto quest'ultimo, soprattutto nel nostro caso, si è dissolto nelle logiche di costruzione intersoggettiva dei testi -, prima come soggetto "rivoluzionario" e poi come soggetto "istituzionale" (MARRONE, 2008), definisce l'identità dei soggetti "presentificati", presupposti e implicati nell'enunciazione del proprio discorso attraverso processi di configurazione della loro immagine visuale (PEZZINI, 2008) e insieme culturale. Semplificando, possiamo dire che prenderemo in analisi le seguenti soggettività inscritte nel Discorso del Comune: l'Amministrazione capitolina, nella posizione di "Enunciatore", il Cittadino, quale "Destinatario" della comunicazione, e infine la città di Roma, come soggetto dei suoi "Enunciati".

Per compiere il nostro cammino di ricerca, procederemo all'analisi semiotica di un corpus di materiali abbastanza eterogenei - ma tutti effetto delle "pratiche di auto-rappresentazione" (SEDDA, 2012) della medesima "Istanza dell'enunciazione" - comprensivo di stemmi, monete, architetture, discorsi politici, testi giuridici, logo, identità visive, autobus e stazioni metro, e lo metteremo in relazione con i grandi eventi della storia di Roma e dei suoi abitanti. Posta una tale varietà di generi testuali dobbiamo a questo punto dare qualche spiegazione a chi, leggendo il titolo di questo articolo, si aspettava l'analisi di un "semplice logo". Ci dispiace deludere le attese di questo lettore, ma l'accezione che diamo alla nozione di "identità visiva" è, al tempo stesso, più profonda e più estesa di quella "comune", e fa riferimento alla definizione che ne dà Jean Marie Floch nel libro ad essa dedicato (1997). Più "profonda" in senso verticale, in quanto tali sistemi di rappresentazione sono assunti come espressioni di un apparato semantico che comprende culture, etiche ed estetiche dell'intersoggettività di riferimento. Più "estesa" sul piano orizzontale, in quanto l'identità visiva non è espressa solo nei "logo", inteso come genere testuale caratterizzato da determinate regole di costruzione retorica, ma in tutto un sistema di "supporti testuali" il cui limite non è mai dato una volta per tutte. Laccezione trova d'altronde giustificazione in un ragione di metodo. Ovvero nel principio epistemologico per cui il significato di un testo non può essere colto se non a partire dalla presa in considerazione e dalla ricostruzione dei testi che sono posti rispetto ad esso in un rapporto di intertestualità. Da qui la necessità di allargare il corpus sino al limite del suo campo intertestuale (limite sempre rinegoziabile) al fine di poterne cogliere tutte le determinazioni semantiche di cui sopra. A questo punto il lettore più avveduto avrà capito che 
lo Stemma, che in apertura abbiamo posto in primo piano, assume per noi una doppia funzione: da una parte lo abbiamo usato come una sorta di "simbolo" della nostra ricerca, dall'altra ci è stato utile come "sistema di riferimento" per orientarci e meglio per rendere conto delle trasformazioni dell' identità visiva e del discorso che essa rappresenta.

Prima di lasciare spazio all'analisi, vogliamo chiudere questa introduzione con una serie di domande che ci hanno accompagnato e guidato durante tutte le fasi della nostra: come le pratiche di autorappresentazione si strutturano in immanenza e come invece sono cambiate nei processi storici? Quali sono i soggetti e gli attori sociali coinvolti nei processi di definizione delle identità che esse attivano? Quali sono le formazioni semiotiche e i linguaggi che esse impiegano? Con quali altri testi occorre metterle in correlazione per spiegarne i processi sottesi? Quali tipi di relazioni esse spengono, attivano o narcotizzano? La nostra dunque è una prospettiva analitica fortemente "relazionalista" (SEDDA, op. cit.) tesa a mostrare come l'iniezione e i "flussi” di testi, figure e immagini in un dato "panorama culturale" (APPADURAI, 1996) muta le morfologie delle strutture semantiche attraverso la trasformazione continua delle relazioni giuntive di termini, idee e soggetti.

\section{Il Comune medioevale. Conquista dell'indipendenza e rimozione dalla storia}

Pratiche di rimozione, operazioni di negazione

Una ricerca che entro una prospettiva diacronica voglia far luce sulle pratiche di rappresentazione simbolica della città, realizzate dagli Enunciatori istituzionali, deve a nostro avviso prendere in considerazione sia i segni che sottendono delle operazioni semiotiche di affermazione dei soggetti storici, sia quelli che, al contrario, in qualche modo implicano o presuppongono una loro negazione. E d'altro canto non potremmo che seguire questa strada: la "negazione" viene definita nel Dizionario di Semiotica come "una delle due funzioni dell'enunciato del fare, [essa] regge gli enunciati di stato operando delle disgiunzioni fra soggetti e oggetti” (GREIMAS e COURTÉS, 2007, p. 220). Da qui, riportando la teoria al nostro caso, l'importanza di spostare la nostra sensibilità proprio sui vuoti della storia, per far emergere non solo i soggetti politici che hanno segnato i destini di intere popolazioni, ma anche le strategie e le dinamiche che si sono susseguite nei loro "rapporti di forza" (DELEUZE, 1986). Come vedremo sono proprio queste operazioni che hanno caratterizzato le pratiche di ri-scrittura della storia di Roma. In tal senso potremmo dire che è proprio in quei luoghi del tempo sui quali la storia pone il velo del silenzio che la semiotica deve recarsi per fare ricerca e analizzare i testi-documento capaci di svelare vecchi segreti o, se si vuole, nuove soggettività.

\section{La storia (mancata) dello stemma}

E' a partire da questa prospettiva che abbiamo inteso approfondire la nostra ricerca, che ha comunque come punto di partenza lo stemma del comune di Roma. Le ricognizioni bibliografiche sul caso ci hanno portato a conoscenza di tre saggi scritti dal romanista Carlo Pietrangeli e pubblicati nella rivista Capitolium (1). Gli articoli sono tutti di grande importanza al fini 
del nostro studio, e in particolare lo sono per due ragioni: la prima è che sono gli unici documenti in cui si tenta di fare uno studio filologico sugli emblemi della Capitale; la seconda è che proprio per questo motivo sono le uniche testimonianze del modo in cui la storiografia ha valorizzato i simboli della città di Roma. O almeno così ci saremo aspettati se non fosse che la loro lettura nonostante sia stata proficua per la quantità di materiali raccolti, nulla ci ha rivelato sul loro valore né tantomeno sul loro contesto storico.

Nel primo dei tre articoli, "Lo stemma del comune di Roma" del 1953, l'autore ordina sull'asse cronologico le diverse varianti dello stemma, a partire da quello che è stato riconosciuto essere il più antico. Nel secondo articolo, pubblicato nello stesso anno, l'autore estende lo studio degli stemmi usati nella Roma medioevale dalle casate dei nobili romani. Infine, nel 1957, Pietrangeli ritornerà sul tema per dedicare un nuovo articolo allo stemma, senza però di nuovo chiarirne il significato né far luce sul periodo storico.

\section{La rimozione dal paesaggio}

In effetti le tracce della Roma di epoca comunale (XI-XIV sec.) oltre ad essere "rare" all'interno del discorso storiografico, sono quasi del tutto nulle nei panorami urbani. Come ha avuto modo di scrivere Jean Maire Vigueur, accademico medioevalista specializzato in storia romana, "il Medioevo è quasi scomparso dal paesaggio romano" (2011, p. XI). Mentre nei paesaggi delle altre città italiane - si pensi, per fare qualche esempio tra i più conosciuti, alle città di Perugia, Siena, Venezia, Firenze - sono ancora presenti i segni lasciati da un epoca comunale fiorente, a Roma niente è in grado di evocare quel periodo. Tra i soggetti che hanno programmaticamente partecipato a questo vero e proprio processo di rimozione della Roma medioevale, dobbiamo annoverare, in ordine cronologico: i papi del rinascimento che hanno voluto una città barocca; lo sventramento che segui all'unità d'Italia per rispondere alle esigenze di una città divenuta Capitale; e infine il fascismo che ha riportato in luce la Roma antica e creato nuovi assi viari (2).

\section{Il discorso storico su Roma medioevale}

Cancellata dal paesaggio, Roma medioevale ha inoltre sofferto a lungo del discredito degli storici che a partire dal Medioevo si sono impegnati nel fornire l'immagine di una città ricoperta di rovine in stato di abbandono, e quasi si compiacevano nell'opporre i vizi dei romani del Medioevo alle virtù eroiche dei loro lontani antenati. Così ancora in tempi recenti gli storici non hanno fatto che consolidare l'immagine di una Roma medioevale in pieno declino che, anche in epoca comunale, si rivela incapace di sottrarsi alla sferza del papato come di conquistare quella piena autonomia senza la quale le altre città dell'Italia dei comuni non avrebbero mai conosciuto il prodigioso sviluppo dei secoli XII, XIII e XIV(ib.).

Certamente non sono mancati i tentativi di separare la storia di Roma da quella dei papi. Esemplare in tal senso è un grande libro di Duprè Theseider (1952), sulla storia di Roma degli anni che vanno dal 1252 al 1378, nel quale l'autore ha compiuto il notevole sforzo di scrivere la storia della città come una storia del comune di Roma e non del papato (3). Nell'opera però, i rapporti 
tra il papato e il comune romano, vennero definiti a partire dalle fonti di archivio, ricche sul versante del papato, che portarono l'autore a esagerare il peso del papa e dell'imperatore e a sottostimare quello del comune e dei romani. Bisogna tener presente a questo proposito che dopo il sacco di Roma, ad opera dei mercenari di Carlo V (soprattutto lanzichenecchi, i quali in gran parte erano luterani ostili ad ogni segno dello sfarzo della Chiesa), dagli archivi del Vaticano sparì quasi ogni traccia del comune di Roma e delle altre istituzioni civili (4).

Dal punto di vista politico quindi, il comune di Roma, che pure è noto per aver governato la città dal 1143 al 1398, non è mai stato considerato dagli studiosi un organismo davvero autonomo e svincolato dalla tutela papale, mentre nel resto dell'Italia comunale la capacità di affrancarsi da qualsiasi autorità esterna al comune, che si trattasse di quella dell'imperatore, del vescovo o delle stirpi signorili, appariva come una delle caratteristiche più sorprendenti di quella civiltà urbana così tipica dell'Italia medioevale (ib., p. XIII). Anche dal punto di vista economico, l'immagine veicolata dagli storici, era quella di una città nella quale i baroni, con la benedizione del papato, erano abbastanza potenti da imporre la loro egemonia sul comune di Roma.

\section{L'altra Roma. Formazioni semiotiche medioevali}

Nel corso degli anni sessanta e settanta vi è però una svolta all'interno del paradigma storiografico, grazie alla scuola delle Annales. Il cambio di rotta prevedeva accanto alla consultazione degli archivi classici l'introduzione dello studio delle strutture economiche e sociali. Il che fu più efficace per l'analisi della storia di Roma in quanto, come abbiamo scritto, gli archivi del comune erano andati persi dopo il sacco del 1527. Questa svolta ha portato gli studiosi, seppure in modo tardivo, a interessarsi della storia della Roma medioevale affascinati dall'analisi di un sistema politico che presenta numerose analogie con le democrazie sia antiche che moderne.

Parallelamente, l'allargamento del corpus delle fonti nell'ambito della storia culturale ha rivelato gusti, interessi e conoscenze dei vari livelli della popolazione dell'epoca. Il nuovo modo di concepire la storia della cultura ha indotto gli specialisti a occuparsi a nuove forme di espressione culturale e a interessarsi alla questione del rapporto della popolazione con il ricordo e le vestigia dell'Antichità classica. Venne così alla luce che ogni romano intratteneva con l'Antichità classica rapporti la cui intensità e natura dipendevano si dal suo livello culturale, ma che in ogni caso contribuivano a rinforzare un'identità comunitaria unica nel suo genere (ib., pp. XV-XVI).

La società era composta non solo dagli strati cosiddetti "popolari", che erano quelli più poveri, ma anche dall'insieme delle categorie socio-professionali - come ad esempio: mercanti, artigiani, uomini d'affari - che nella scala sociale si collocavano appena al di sotto della nobiltà. E' stata quest'ultima parte di popolazione che, nel 1143, aveva preso l'iniziativa di fondare il comune e aveva fornito i "cavalieri" indispensabili alla difesa della città (i milites, da cui l'appellativo di militia che viene attribuito alla nobiltà cittadina). Sul piano dell'identità di gruppo questa classe traeva la sua coesione dalla pratica 
del combattimento a cavallo e dal fatto che i suoi membri aderivano ad un sistema di valori fondato sull'esaltazione del lignaggio ed un uso controllato della violenza. Da punto di vista politico è quindi a questa classe che si deve la creazione del comune, il quale è stato preceduto da molteplici iniziative provenienti dalla militia, che esprimevano la volontà di emanciparsi dalla tutela pontificia. Fino alla metà del XIII secolo questa classe sociale riesce a monopolizzare le cariche più importati, consentendo al contempo di far entrare alcuni membri del popolo. Più che dal popolo, infatti, il pericolo per l'egemonia veniva da un ristretto numero di famiglie che, grazie ad una forsennata pratica nepotistica da parte dei papi, riesce nel giro di tre generazioni a prendersi le più alte cariche comunali. E' questa la classe dei baroni, il cui stile di vita diverge sotto molti aspetti da quella della militia. Infatti, mentre quest'ultima deve il suo potere e prestigio dalla rendita fondiaria e dagli affitti convenienti dalle chiese romane, i baroni lo devono alle attività economiche presso i villaggi fortificati.

\section{L'isomorfismo tra classi sociali e forme di governo}

Lo sguardo sulle classi sociali romane è tanto più importante se si pensa che le relazioni tra le configurazioni dei gruppi sociali che salgono al potere e le forme di governo che essi producono e inventano, si determinano entro un regime di isomorfismo strutturale. Ciò in altri termini significa che le forme di governo assunte dal comune riflettevano e si adattavano perfettamente al profilo della classe che deteneva il potere. Nel corso del primo periodo (11431250) il senato è composto da diverse decine di membri che riflette il carattere relativamente egualitario che si diede la classe della militia. Nel corso del secondo periodo (1250-1350) il potere è nelle mani di due senatori scelti tra le famiglie dei baroni (Orsini e Colonna). Nell'ultimo periodo (1350-1398) il popolo esercita il potere attraverso due differenti organismi: una magistratura collegiale - composta da sette riformatori e tre conservatori -, nella quale la nobiltà poteva accedere in posizione minoritaria, e un organismo esterno, la Felice società dei balestrieri e dei pavesati, sorta di partito politico, composto da seguaci del popolo, che svolgeva la funzione di controllo dell'attività comunale. L'egemonia dei baroni non ha impedito ai romani di dotarsi di dodici diverse forme di governo (dalle più collegiali a quelle più autoritarie). Solo questo aspetto rivela la grande capacità di dinamismo e di adattarsi alle esigenze del momento che ebbero i romani nel Medioevo.

L'esperienza del comune durerà sino al 1398 quando, nel luglio di quell'anno, incapace di superare la divisioni interne, abdicò tutto il potere nelle mani di Bonifacio IX, un papa che aveva fatto della restaurazione dell'autorità pontificia il suo programma e che non avrà alcuno scrupolo a reprimere ogni successivo tentativo di emancipazione da parte dei romani.

\section{La rivoluzione del 1143 e la "renovatio senatus"}

Jean Maire Vigueur ha definito il colpo di stato del luglio 1143 uno degli: 
1870, mise fine a quasi cinque secoli di dominio pontificio sulla città (ib., p. 259).

Le poche informazioni che ci sono arrivate sullo sconvolgimento di cui Roma fu teatro nel corso del 1143, raccolte dalla cronaca scritta da Ottone di Frisinga, zio del futuro imperatore Federico Barbarossa, ci raccontano che i romani, dopo vari tentativi, ad un certo punto, presero d'assalto il Campidoglio e procedettero alla Renovatio senatus.

La scelta di assegnare al nascente soggetto politico il nome che apparteneva alla forma di governo vigente nel periodo della Repubblica, Senatus, da un punto di vista semiotico non era certamente neutrale. Infatti assegnare tale denominazione alla neonata assemblea cittadina significava, per i romani, ridare vita all'istituzione che incarnava la grandezza della repubblica romana e con essa ri-attualizzare i valori negati dalla storia. Ma al tempo stesso, ridare vita al senato significava intervenire sui confini della città, spostandoli il più lontano possibile, e con essi l'ampiezza del territorio sul quale il senato poteva esercitare il proprio potere. Insomma, tale denominazione serviva in un certo qual modo a legittimare le azioni militari attuate nella prima fase storica del comune, che lo vede impegnato ad annientare le città (Tivoli in primis) e borghi (specialmente Tuscolo e Albano) che si contendevano il controllo sulla campagna romana (ib. p. 267).

D'altra parte, la restaurazione del senato, la scelta generale di far richiamo esplicito ai simboli della Repubblica, andava a incidere sui rapporti di potere non solo con lo Stato della Chiesa ma anche con l'Impero. E' nella seconda cronaca di Ottone di Frisinga, dedicata ai primi anni del potere di suo nipote Federico Barbarossa, che si trova traccia di una lettera del 1149 indirizzata all'imperatore Corrado III, nella quale l'utilizzo da parte del senato della formula Senatus Populusque Romanus lascia intendere che quest'ultimo si considera erede e continuatore del Senato antico, e cioè della gloriosa istituzione che aveva fatto grande l'impero della Repubblica. I senatori, lamentandosi della mancata risposta, si prodigarono nel rimembrare all'Imperatore che grazie al restauro del senato l'impero poteva ritornare ai fasti del passato. Ma Corrado, al tentativo dei romani di sottrarre dalle mani della Chiesa lo strumento dell'assenso all'elezione dell'Imperatore, rispose rifiutandosi di diventare ago della bilancia tra i Romani e il Papato, preferendo infine la via dell'intesa con i pontefici (cfr. GATTO, 1999, p. 336). Quello che i romani non menzionarono in queste prime fasi, ma che più tardi, nel 1346, Cola di Rienzo non lasciò sottinteso, è che è il senato a nominare l'imperatore, così come voluto dalla lex regi (5).

Dinamiche storiche. Le frizioni tra i romani e il papato per il dominio su Roma

In realtà la rivoluzione del 1143 non fu altro che l'esplosione di un 
processo graduale (LOTMAN, 1994) che vedeva i romani nel tentativo di sottrarsi all'autorità pontificia. I romani sin dal XII secolo avevano intrapreso una politica di espansione che era contraria agli interessi del papato e che portava spesso queste due entità ad entrare in conflitto. L'evento che accelerò in maniera esplosiva il conflitto fu in occasione di un intervento di Innocenzo II mirato a privare i romani dei frutti della vittoria riportata sugli abitanti di Tivoli, vittoria che arriva dopo due anni di guerra per il controllo della Campagna romana. E' chiaro dunque che nel territorio romano oltre all'esercito pontificio, erano attive da tempo le truppe della nobiltà romana. Non bisogna dimenticare che, come abbiamo accennato, la nobiltà romana si era data un'identità di gruppo molto forte, che li aveva già spinti a organizzarsi sul piano politico fondando sedici anni prima un consiglio formato da sessanta senatori. E' quello che fa parte della preistoria del comune, una fase di latenza durante la quale l'organizzazione conobbe un periodo di esistenza intermittente, che la accomuna alla storia degli altri comuni italiani (ib., p. 261).

Il conflitto simbolico tra il Campidoglio e il Laterano.

Il gesto del 1143 colpisce più per la portata simbolica che non per la violenza, perché non è ancora assodato se i romani per impadronirsi del Campidoglio abbiano fatto ricorso alla forza. Tanto più che al tempo il colle aveva solo un valore simbolico e per questo motivo probabilmente niente impedì ai romani di salirci sopra e, in un unico gesto, di rifondare il senato. Il fatto che fosse un luogo con valenza simbolica non frenò poi i romani nell'innalzarvi la loro sede politica e seguentemente di fortificarla. Fortificazione che servì loro per difendersi da una truppa inviata dal re di Sicilia, unico tentativo da parte del papato di riprendersi con la forza il possesso del Campidoglio, che da lì in poi diverrà il simbolo dell'indipendenza comunale.

L'attuale palazzo del Campidoglio, che ospita tutt'oggi l'istituzione erede del comune medioevale, sembra non presentare segni di epoca medioevale, se non per la torre che rompe con il resto dell'edificio (fig.1).

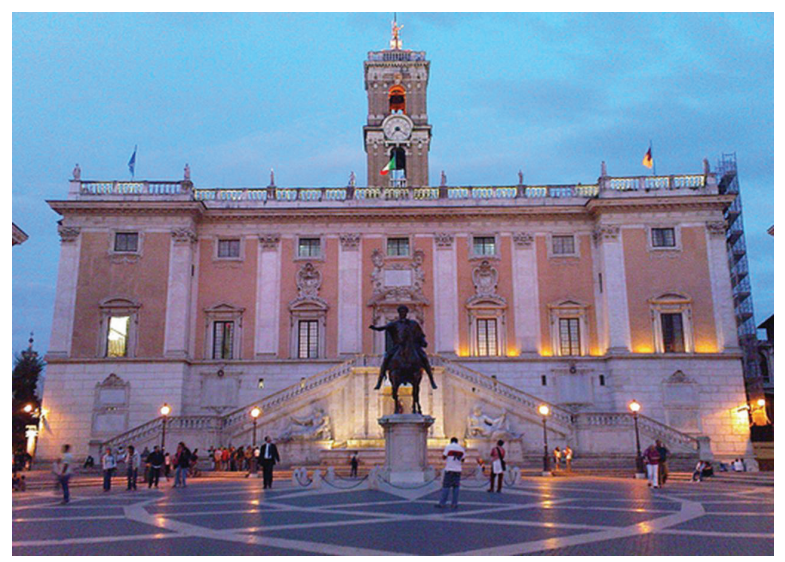

Attuale facciata del Campidoglio. 
Ma a ben guardare, al di sotto della splendida facciata disegnata da Michelangelo, sono conservate quasi tutte le strutture medioevali. E' partendo da questi segni e unendoli ad altre testimonianze, che gli storici hanno potuto ricostruire il Palazzo così come doveva presentarsi in epoca medioevale (fig. 2), intorno alla metà del XIII secolo ( ib., p. 357).

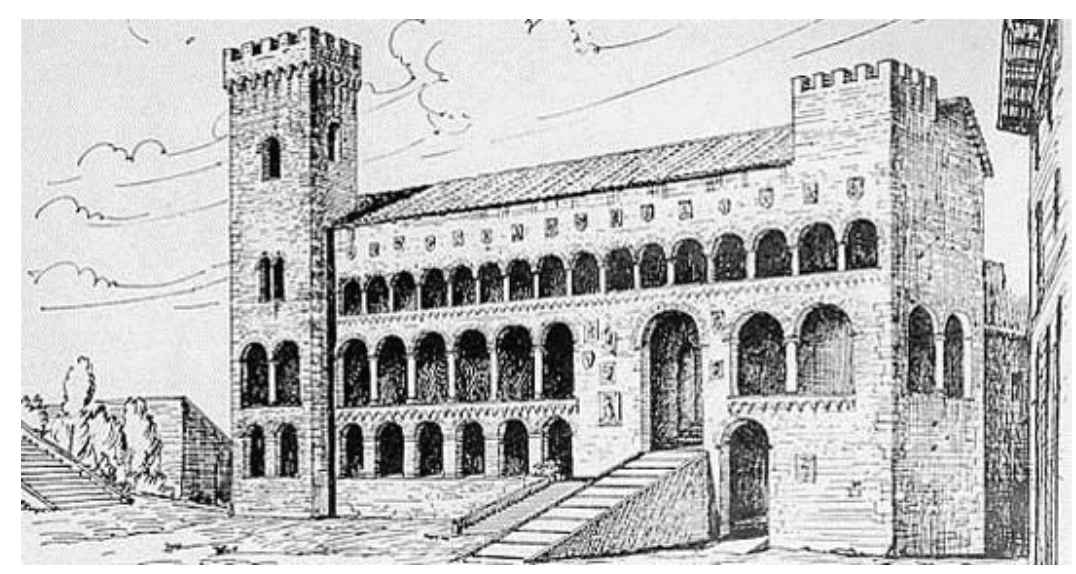

Palazzo senatorio, metà del XIII secolo.

Più difficile sembra la ricostruzione dell'edifico così come doveva essere a partire dal 1143 . Un dato importante è che i senatori fecero ricostruire l'edificio in modo tale che girasse le spalle al Foro e guardasse verso la parte occidentale della città, quella compresa nell'ansa del Tevere dove si concentrava la maggior parte della popolazione. Il cambio di orientamento era in realtà un'operazione semiotica funzionale a mettere in relazione il nascente comune con il popolo che aspirava a governare. Da quel momento e per tutta l'epoca medioevale durante il quale Roma era governata dal suo comune, la piazza sulla quale si affaccia il palazzo comunale diventa sede dell'arenga, cioè l'assemblea di tutti i civies, e si afferma allo stesso tempo come luogo in cui, sino ad epoca comunale, i romani si raduneranno non solo per partecipare alle riunioni del parlamento, ma anche per manifestare, protestare, fare pressione sui magistrati e i membri che occupavano le più alte cariche del palazzo comunale.

Il Campidoglio era un luogo che ai romani doveva apparire di fortissima valenza simbolica, motivo per cui i senatori si sforzeranno per portarlo alla bellezza delle sedi del potere del papato: il Laterano e il Vaticano. E' mettendo in comparazione questi punti emblematici del potere che si comprende il senso delle opere e delle decorazioni che li riguarderanno. In evidente competizione con il Vaticano, dove i romani potevano ammirare l'unico obelisco ancora in piedi, sulla piazza del Campidoglio il senato fa erigere un obelisco che poggia su quattro leoni di pietra, simbolo del popolo romano durante la repubblica e del potere giudiziario nel periodo medioevale. E ancora, così come sulla piazza del Laterano era stata collocata la celebre statua del Marco Aurelio, che al tempo si pensava raffigurasse Costantino, sulla piazza del Campidoglio i senatori espongono un'altra scultura antica, anch'essa richiamante i 
valori della repubblica, ma rispetto alla quale il comune rivendicava il monopolio, dichiarandosi l'unico erede legittimo.

A questo si devono aggiungere i lavori intrapresi a metà del XIII secolo per ingrandire e rinnovare il palazzo che si inquadrano in questa politica. Il nuovo palazzo è costruito sui resti del tabolarium, ma la novità è che la facciata guadagna una buona decina di metri. Il nuovo palazzo è alto tre piani e ogni piano utilizzava colonne di reimpiego di tipo ionico. Michelangelo si è ben guardato dal toccare le colonne che sosteneva le arcate della loggia, cosicché è possibile vederle ancora oggi a patto di essere ammessi alla sala del consiglio (6).

\section{Lo stemma del Comune}

Forti del nostro percorso di ricerca sulla storia, la società e la cultura medioevale romana possiamo ora prendere in carico i documenti storici relativi allo stemma per rendere conto del suo significato e valore mettendolo in relazione con le altre serie, simboli e pratiche che, come abbiamo visto, hanno partecipato alla costruzione semiotica del comune di Roma. D’altronde, come scrive Franciscu Sedda:

Capire la storia e il valore di un simbolo significa avere sensibilità per le relazioni
interne - il simbolo con le sue componenti plastiche e figurative - e per quelle
esterne - il simbolo in rapporto agli altri simboli e alle altre serie (materiali,
economiche, politiche, istituzionali, intellettuali, artistiche...) che compongono
e strutturano, benché sempre in modo incompleto, la vita sociale (SEDDA, 2012,
p. 363).

E' grazie alle ricerche dello storico romanista Carlo Pietrangeli che siamo a conoscenza del simbolo scelto dai senatori medioevali per definire la propria identità (fig. 3). Ed ecco come lo storico procede alla sua descrizione:

Scudo a capo piano, fianchi verticali e piede ritondato, blasonato di rosso con la crocetta d'argento e con le lettere SPQR poste verticali e discendenti a banda. Un ornato a mosaico racchiuso fra modanature incornicia lo scudo, forse riproducendo schematicamente la bordura inchiavata che doveva esistere nei più antichi stemmi della città (PIETRANGELI, 1953a, p. 58) (7).

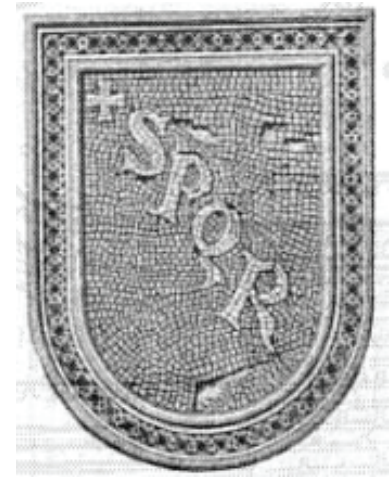

Primo stemma del comune di Roma. 
Lo stemma dunque riporta nel proprio campo l'acronimo della formula repubblicana Senatus et PolulosQue Romanus. La croce che precede l'acronimo non fa che confermare la volontà del Comune di annettere sotto la propria giurisdizione tutti i territori che facevano parte dello Stato Romano, dentro il quale era pure presente lo Stato della Chiesa.

Per quanto riguarda i colori utilizzati si può notare come in realtà il loro utilizzo risale ai secoli dell'impero romano quando, lo stesso esercito era solito portare il vessillo rosso porpora raffigurante l'aquila con l'acronimo SPQR scritto in oro. L'oro raffigurava il sole e la luce, mentre il rosso era il colore di Marte dio della guerra (PIETRANGELI, op. cit.).

Dobbiamo poi far presente che la scelta del supporto testuale (FONTANILLE, 2008) non fu per niente casuale o priva di significato. I mosaici infatti erano più preziosi delle altre forme di decorazione, come per esempio l'affresco, per via dei materiali utilizzati e dei costi dovuti alle tecniche di esecuzione. Per queste ragioni e per il fatto che il mosaico resiste meglio all'usura del tempo, esso ispira come un timore reverenziale che gli assicura una sorta di perennità. Basti pensare tra l'altro che questa tecnica di decorazione nella tradizione cristiana, a partire dal IV secolo, non ha lo scopo di raccontare storie ma quello di manifestare la verità della fede, a cominciare dalla maestà divina e dalla missione salvifica degli apostoli, dei santi e degli altri personaggi dotati di un'autorità particolare, come il papa o l'imperatore (8) (cf. VIGUEUR, 2011, p. 345).

Successivamente lo stemma sarà soggetto di variazioni plastiche in particolare cambierà la forma dello scudo che dal modella a piede ritondato passerà al tipo "gotico" più stretto e con piede a punta - che ai fini del nostro lavoro è inutile approfondire in questa sede. Ci sembra invece interessante l'aggiunta di una importante componente figurativa, la corona, che significava la conquistata sovranità dell'entità politica, la cui testimonianza più antica risale al secondo quarto del sec. XIV e fu rinvenuta nella "rugitella del grano" (fig. 4) (9).

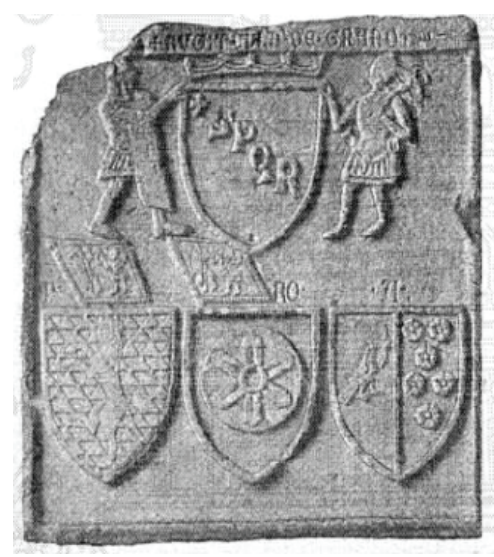

Rugitella del grano (sec. XIV). 
Sulla tavola marmorea infatti sono presenti gli stemmi usati a Roma sotto le reggenze popolari, e tra questi troviamo lo scudo con la scritta SPQR. L'uso della corona sugli stemmi risale quindi ad un periodo di governo popolare, durante il quale doveva rappresentare l'indipendenza e la libertà del popolo romano. Nasce così il nuovo simbolo della milizia cittadina che viene affisso in Campidoglio (PIETRANGELI, op. cit.). C sembra importante menzionare questa variazione e mostrare questo documento anche perché, come vedremo, la configurazione dello scudo presenta notevoli somiglianze con quella dello stemma attualmente impiegato dall'Amministrazione capitolina:

\section{La monetazione}

Il riferimento ai valori e alle credenze dominanti durante il periodo della Roma antica fu una scelta strategica che i Romani adottarono anche nella monetazione. Esemplare a riguardo è la serie di monete risalenti al 1200, le quali recavano la raffigurazione della dea Roma che, per tutto il periodo dell'età della Repubblica e dell'Impero romano, era stato il simbolo con il quale veniva identificata la città. L'immagine della dea, infatti appare sin dal II secolo a.C. proprio nel denario romano, nel quale viene raffigurata con il capo di profilo e cinto da un elmo (fig. 5).

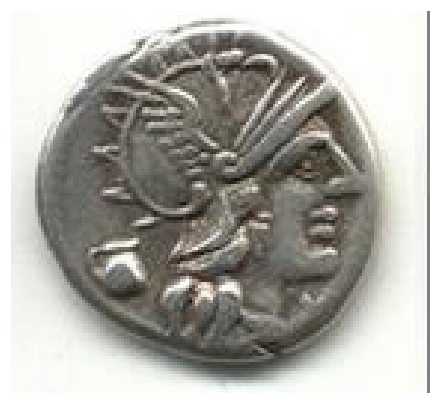

Denario romano del II secolo a.C..

Se pure non è nostro compito qui estendere le analisi al periodo della Roma antica, ai fini del nostro studio possiamo perlomeno ipotizzare che l'impiego della dea nella monetazione del tempo partecipava ad una narrazione che sosteneva la verità di una identificazione del potere politico con quello religioso. Ma se questa rappresentazione poteva apparire naturale agli occhi dei romani di epoca imperiale, un po' meno doveva esserlo a quelli dell'epoca dei comuni. O perlomeno, la riabilitazione della dea che apparteneva all'impianto mitologico di epoca classica, non poteva che apparire come una provocazione agli occhi del papato.

Ciò è ancor più valido se si considera l'altra figura impiegata nella serie di monete coniate dal senato romano nel 1200 (fig. 6).

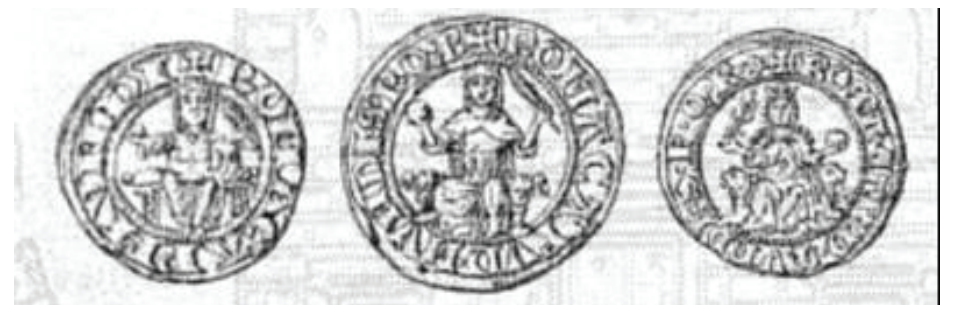

Monete recanti la legenda Roma caput mundi, l'acronimo Spqr e la croce. 
Nelle monete infatti, seduti ai piedi della dea, la quale come abbiamo detto rappresentava la città e l'istituzione che la governava, vengono raffigurati due leoni, figura quest'ultima che nella simbologia romana rappresentava il Popolo romano (10). Il comune in questo modo, oltre ad auto-rappresentarsi come l'erede dell'impero, adottandone la simbologia, rappresentava i suoi cittadini come i discendenti del popolo romano, e istituiva così una continuità istituzionale tra il sistema di potere della Roma antica e quello della Roma medioevale.

La leggenda presente nelle serie di monete traduce l'impianto simbolico in quello verbale: alla Roma caput mundi, nome con cui si designa la dea, segue l'acronimo del motto della Repubblica Senatus et populosque romanus. Inoltre, rispetto ai rapporti di forza con il Sacro Romano Impero, la riabilitazione della dea doveva servire al Senato riaffermare quella centralità politica che la città aveva perso con la fine dell'impero romano.

\section{Una passione civica: il rapporto con l'Antichità}

Il reimpiego dei simboli dell'antichità per la composizione dello stemma del comune trova un'interessante analogia, di forte valore euristico, con il reimpiego sistematico di colonne, capitelli e mille altri frammenti provenienti da monumenti antichi, per la ristrutturazione delle chiese, edifici e palazzi medioevale di epoca comunale. Tale reimpiego lungi dal rispondere a una preoccupazione economica, obbedisce in effetti a considerazioni di natura ideologica ed esprime la volontà dei committenti di ritornare all'Antichità classica utilizzandone i materiali (testuali, diremmo noi) (cfr. VIGOEUR, 2011, p. 337).Un testo esemplare in materia di reimpiego dell'antichità per la decorazione dei palazzi, è l'abitazione nota con il nome di Casa dei Crescenzi (fig. 7), la quale apparteneva a Nicholaus Centii, membro del senato nel 1163 .

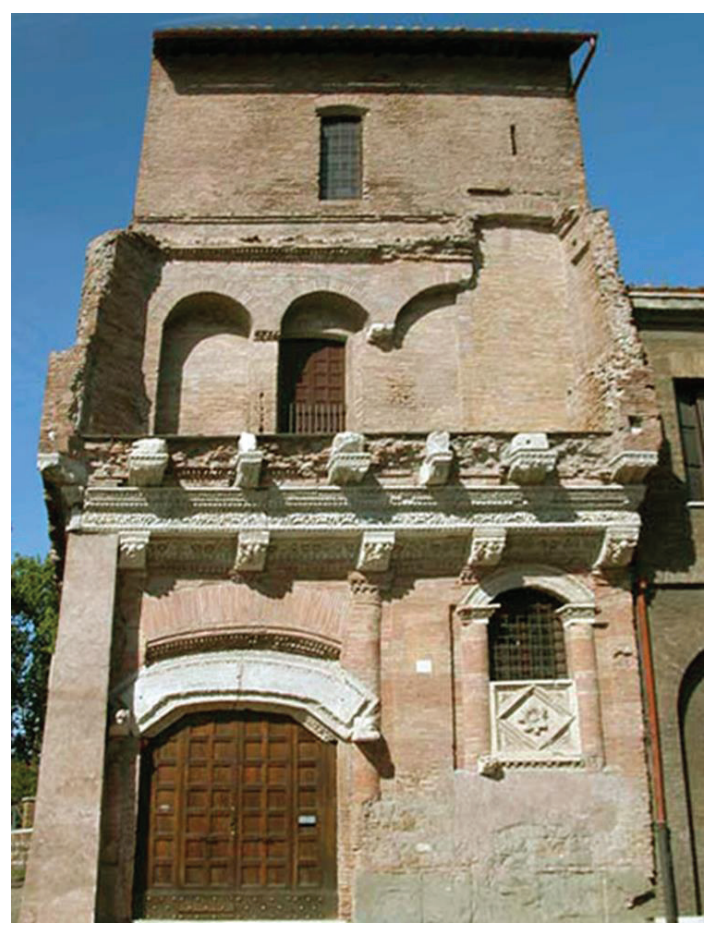

Facciata casa Crescenzi. 
Questo edificio, esemplare unico a Roma, sembra essere stato costruito per poter manifestare la passione del proprietario per la Roma antica. Passione che si carica di connotazioni tanto politiche quanto estetiche. Il profilo dell'edificio è quello di una casa torre molto massiccia. La facciata laterale sinistra ingloba una serie di finte colonne che evocano un tempio pseudo-periptero; il portale è sormontato da una lunga iscrizione nella quale si esprime il desiderio di tornare alla bellezza della Roma antica. I marmorari hanno utilizzato quasi esclusivamente pezzi di sculture antiche, in modo da formare un enorme trabeazione.
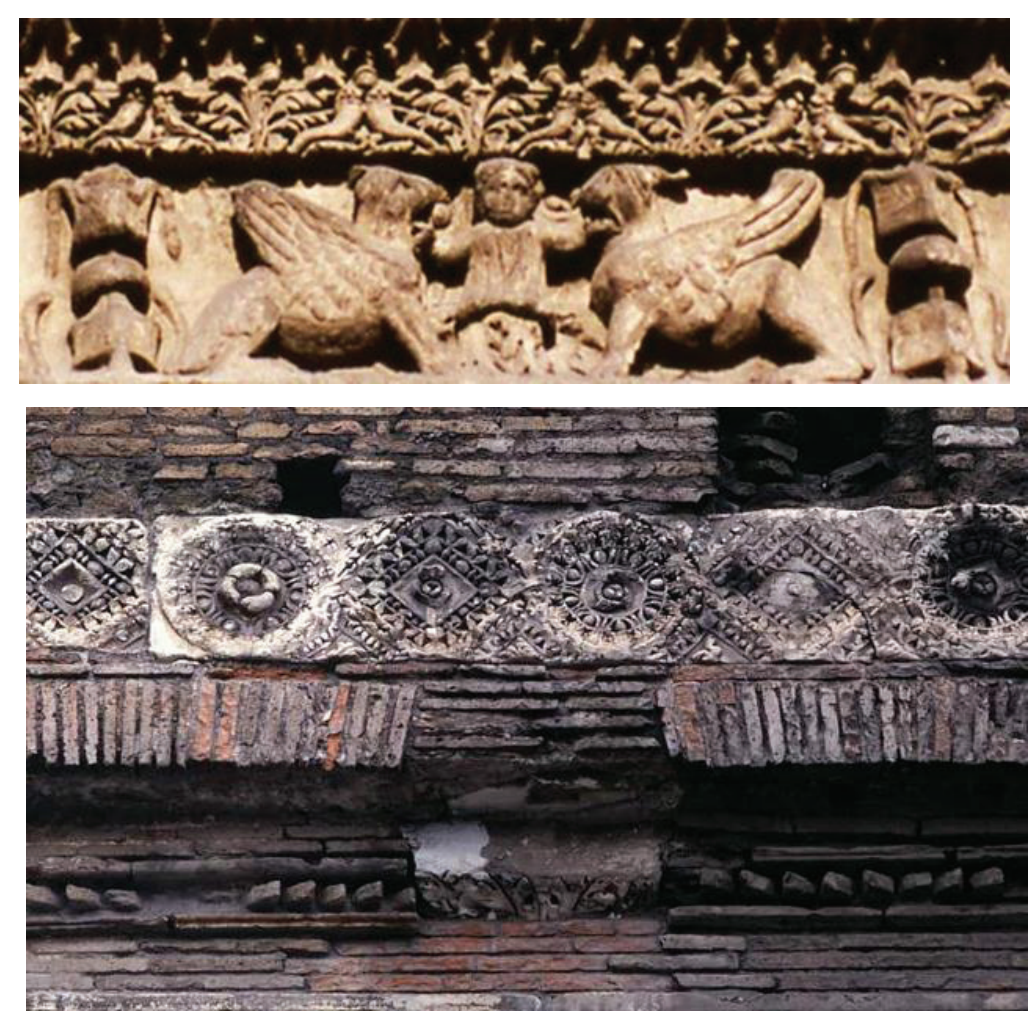

Particolari casa Crescenzi.

Quanto ai piani superiori, a giudicare dai frammenti ancora incastrati nel muro di mattoni, sembra sia stata costellata di blocchi di reimpiego, la cui funzione era più decorativa che statica. Per concludere sembra che il proprietario abbia fatto una questione d'onore l'utilizzare soltanto pezzi di comprovata origine antica (cf. ib. p. 356) (11).

\section{Il mito di Roma e la fine del comune libero}

Il corpus testuale che abbiamo sinora proposto mostra che la relazione dei romani con il proprio passato è fondamentale per comprendere la loro cultura. L'influenza del passato antico si è fatto sentire a Roma in ogni epoca e quanto ne sopravviveva contribuiva a costituire il mito di una forma di vita superiore per la quale i romani provavano un sentimento misto di orgoglio e rimpianto. In ambito politico la lotta tra i poteri per contendersi l'eredità dell'impero era legato ad una idea di perfezione del suo sistema di governo e della sua organizzazione amministrativa. Il passato per la città, le istituzioni e i cittadini romani, non si 
riduceva alla dimensione del ricordo storico, ma ad una realtà suscettibile di diventare attuale non appena si ripresentassero le condizioni favorevoli. Un passato ancora vivo, come la brace che cova sotto le ceneri, sul quale si basavano le speranze rianimare una civiltà in declino (VAUCHEZ, 2001, p. XX). Il desiderio di riallacciarsi a quel brillante riferimento, come abbiamo mostrato, si manifestava non solo nella vita politica ma anche in quella civica. Ciò sul piano politico spiega il successo di espressioni d'ordine programmatico come Renovatio senatus che le altre formazioni semiotiche prendevano in carico per tradurle e riaffermarle in altri linguaggi: gesti, immagini, forme architettoniche fortemente impregnati di suggestioni dell'antichità entrano di prepotenza nell'immaginario collettivo romano, irrobustendo una tradizione politica che non sa e che non vuole rinunziare al mito.

D'altra parte, come ha mostrato Sedda studiando la storia della cultura dei sardi, il mito delle origini produce nella coscienza una concezione del progresso collettivo solo come impossibile ritorno alla perfezione, reale o presunta, delle origini. Per quanto i consoli romani non smisero mai di credere alla possibilità di una renovatio senatus la sua reale attuazione non poté avvenire in quanto il contesto era profondamente cambiato e non si potevano cancellare con un tratto di penna i secoli che li separavano dall'Antichità.

Il mito medioevale di Roma durò per i tre secoli in cui il Comune ha goduto di una autonomia giurisdizionale e segnò le lotte tra le forze che si rivendicavano “romane”. Esso concluse la sua carriera nel 1354 con la morte di Cola di Rienzo e l'allontanamento da Roma di quei poteri che fino ad allora vi avevano costruito la loro sede, lasciando così una città divisa dalle faide tra i baroni e troppo debole per impadronirsi del suo destino.

\section{Il tradimento della memoria}

Con il ritorno dei papi da Avignone e il definitivo reinsediamento della curia romana ad opera di Martino V, lo Stato della Chiesa cominciò a riaffermare e consolidare progressivamente il proprio potere (12) (Miglio 2001, p. 336). La strategia che seguirono i pontefici fu quella di sovrapporre i propri modelli a quelli comunali. A riguardo, la moneta coniata da papa Martino V (fig. 10) è un ottimo esempio di come il papato si rapportò al comune, inglobandone i simboli entro i propri domini.

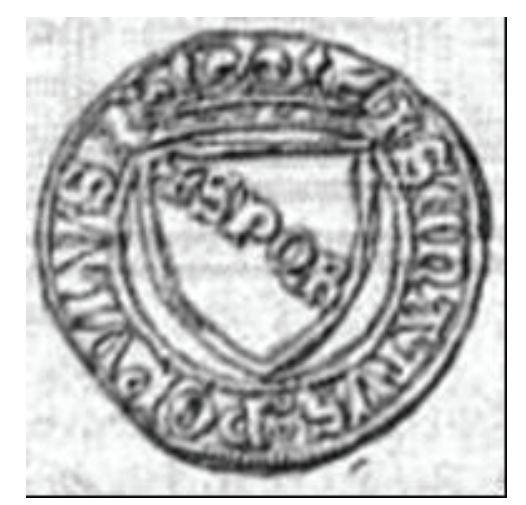

Moneta coniata durante il pontificato di Papa Martino V (1417). 
Inoltre è importante sottolineare come anche lo scudo raffigurato su questa monete sia sormontato ancora dalla corona; ciò testimonia che Papa Martino V durante il suo pontificato ha in effetti riconoscito l'autorità politica e la sovranità del popolo romano sulla città.

L'emblema con la corona cessa di essere utilizzato quando il successore di Martino V, papa Eugenio IV, toglie al Senato di Roma, e quindi al popolo romano, l'autorità giuridica. Questo momento trova testimonianza in un sigillo del 1457 nel quale appare lo scudo senza la corona (fig. 11).

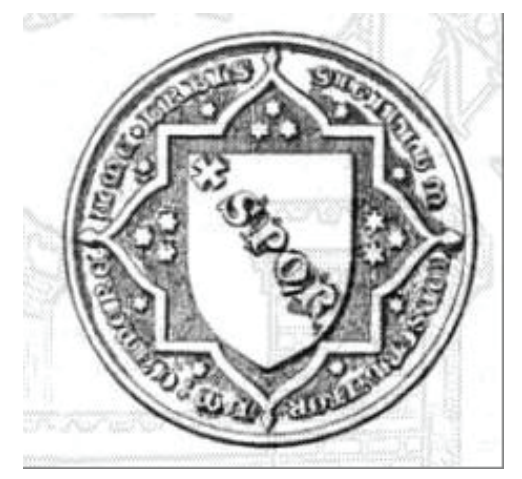

Stemma di Roma durante il pontificato di Papa Eugenio IV

D'altronde il papato non accetto più un'istituzione politica autonoma rispetto al suo potere. Esemplare è in proposito il lento processo di rimozione dal Campidoglio di tutti i simboli della giurisdizione municipale che avevano caratterizzato questo luogo sin dalla nascita del comune, compreso il suo stemma.

L'effetto finale fu quello di imprigionare la coscienza politica comunale dei romani dentro una dimensione puramente passionale, mista di paura e di nostalgia, che ha decretato la definitiva rinuncia ad una qualsiasi azione di rinascita tesa a riconquistare l'indipendenza.

\section{La rinascita del Comune nella modernità}

Facciamo ora un salto lungo cinque secoli per riatterrare nel momento storico in cui il comune di Roma passò dalla sottomissione al potere dello stato della chiesa alla giurisdizione dello stato italiano. Lo faremo continuando a prendere in esame i suoi simboli e in particolare l'evoluzione del suo stemma per tentare di comprendere come si sono gestiti i rapporti di forza con gli altri poteri che hanno segnato l'identità della città.

A causa delle numerose varianti (13), presenti ancora attorno alla fine del XIX secolo, il sindaco Torlonia, nel 1884, nomina una commissione per la determinazione dello stemma di Roma. La commissione, con una relazione datata 18 aprile 1884, presenta il nuovo stemma proposto, che in realtà non fu mai utilizzato poiché quello che apparve nei successivi documenti pubblici è uno stemma che si rifa decisamente a quello presente sulla Rugitella del grano. In araldica questo stemma sarà cosi definito: "di rosso alle lettere in capitale romana SPQR poste in banda, accompagnate nel cantone destro dal capo della croce greca il tutto d'oro, cimato da corona di otto fioroni d'oro, cinque visibili" (Pietrangeli 1957a, p. 57). 
Lo stemma così definito resterà invariato sino ai giorni nostri, tranne per due modifiche imposte dal regime fascista. Infatti con il decreto del 26 agosto 1927, nella parte finale dello stemma viene inizialmente inserito il fascio littorio che, con un successivo decreto datato 12 ottobre 1933, verrà sostituito con il Capo Littorio, inserito nella parte superiore dello scudo di Roma.

In seguito alla caduta del regime, e con la nomina a sindaco di Filippo Andrea Doria Pamphili, nel 1944 viene ripristinato lo stemma di Roma utilizzato prima del 1927, che da quella data in poi resterà uguale fino ai giorni nostri, quando dallo stemma si è passati alla creazione e all'utilizzo del nuovo logo (fig. 12).

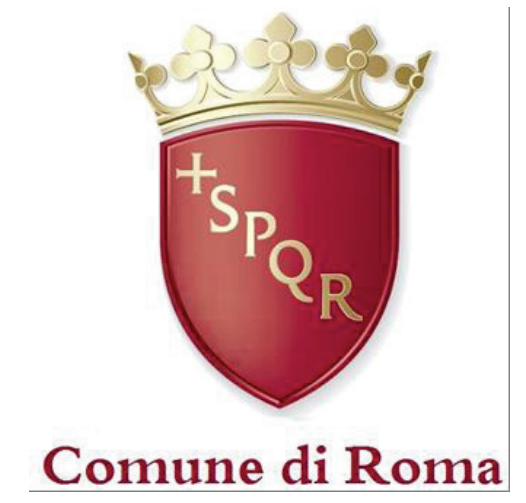

Logo del Comune di Roma (l'ultima variazione che precede la nuova identità visiva).

Con ciò non vogliamo sostenere che il logo nel corso dei decenni non sia stato oggetto di variazioni. Quello che abbiamo riportato in figura non è altro che l'ultimo logo impiegato. Le variazioni di stile hanno interessato solo alcune delle componenti plastiche dell'immagine, andando a cambiare di volta in volta la resa e o gli effetti grafici del logo, con l'effetto di renderlo più attuale rispetto alle innovazioni tecnologiche che si sono susseguite negli anni, e più in linea con quello che si riteneva fosse il gusto e la sensibilità momento. Sarebbe però riduttivo interpretare queste variazioni unicamente come un segno del mutamento del gusto estetico relativo alle forme della cosiddetta "comunicazione istituzionale". Piuttosto, a nostro modo di vedere, quello che le variazioni rivelano è una sempre rinnovata - se mai ce ne fosse stato bisogno - attenzione per le "forme di comunicazione" ed evidentemente dell'importanza che questa riveste per l'enunciatore istituzionale. Lutilizzo delle variazioni permette anzitutto al Comune di presentare se stesso, quindi di autorappresentarsi, come un ente vivo, dinamico, le cui forme mutano con il divenire del mutamento sociale. Per questo motivo ci sembra interessante proseguire il nostro studio presentando alcune delle trasformazioni più rilevanti che hanno riguardato l'identità visiva dell'amministrazione capitolina, cercando di interrogarci sul loro significato.

\section{Da Comune a Capitale. Identità visiva e definizioni}

Con l'entrata in vigore del decreto legislativo attuativo del 3 ottobre 2010 (14), Roma è passata da "città Comune" a "città Capitale". Insieme alla trasformazione che ha riguardato lo statuto giuridico della città e i poteri assunti dall'amministrazione pubblica, è stata oggetto di una profonda ridefinizione anche 
l'identità visiva, della quale riportiamo in figura il nuovo logotipo che ne condensa forme e colori (fig. 13).

\section{roma CAPITALE}

Figura 13. Logotipo Roma Capitale.

Come si può osservare dall'immagine il logotipo conserva la presenza dello storico stemma del comune di Roma. Quest'ultimo, nelle sue componenti principali, non poteva essere cambiato se non andando a ritoccare il primo articolo dello statuto del comune, dove sono indicate le norme araldiche che lo riguardano: "L'emblema del Comune è costituito da uno scudo di forma appuntata, di colore porpora con croce greca d'oro, collocata in capo a destra, seguita dalle lettere maiuscole d'oro S.P.Q.R. poste in banda e scalinate, cimato di corona di otto fioroni d'oro, cinque dei quali visibili" (Statuto di Roma Capitale, I cap., art. I).

Una breve disamina comparativa del vecchio logo con il nuovo, oltre alla presenza della nuova denominazione assegnata all'istituzione preposta all'amministrazione della città, mette in evidenza che i suoi componenti sono stati oggetto di un gioco di inversioni, che hanno coinvolto gli assi verticale/orizzontale e la categoria topologica minore/maggiore. La denominazione "Roma Capitale" rispetto al vecchio logo, nel quale la sigla "Comune di Roma" era stata collocata nella sua parte inferiore, appena sotto lo stemma, ora segue quest'ultimo sul piano orizzontale, e contrariamente al passato è ora la sigla a coprire una porzione maggiore di spazio, risultando così più visibile e certamente predominante nell'economia testuale.

Rimanendo sulla componente verbale del testo, che riporta il nuovo nome assegnato all'attore istituzionale, dobbiamo soffermarci sullo stile del carattere tipografico impiegato. Questo, denominato "Urbs", è stato creato ad hoc da Inarea, l'agenzia di comunicazione che ha realizzato la nuova identità visiva del comune. Secondo le volontà dei creativi che ne hanno curato l'ideazione, il neonato carattere nasce dalla "sovrapposizione" tra lo stile della scrittura rinascimentale del XV secolo e quello impiegato nell'antichità per le epigrafi. Quest'ultimo è il carattere lapidario romano, utilizzato nel vecchio logo per l'acronimo S.P.Q.R. presente all'interno dello scudo (15). Rispetto alle forme plastiche del carattere Urbs, si può notare la presenza di linee caratterizzate da contorni netti, curve dolci, dall'alternanza di tratti sottili e spessi, da angoli netti e dalla presenza delle cosiddette "grazie", ossia da allungamenti presenti nella parte finale di alcune lettere.

E' con l'aggiunta di questi tratti che il carattere Urbs tenta di dribblare e superare il Lapidarium. Ma lo fa riproponendo il vecchio per cosi dire "aggraziato" 
con i tratti dello stile rinascimentale.

La creazione del nuovo carattere "dedicato" esprime quindi una innovazione creativa che sovrapponendo stili ma soprattutto epoche molto lontane tra loro richiama quel particolare rapporto con l'antico proprio della cultura medioevale. Ovvero la tendenza a costruire a partire dalle macerie del passato, creando stratificazioni o ibridazioni stilistiche, che hanno l'effetto di attivare relazioni giuntive tra momenti storici molto diversi tra loro, e tra il passato e il presente. Una logica che al suo estremo contiene in nuce la stessa idea che stava alla base della Renovatio, cioè che non si possa pensare una nuova identità se non come restaurazione di quella passata.

Per l'analisi semantica del lessema "Capitale", che sotto il rispetto del diritto amministrativo qualifica lo statuto giuridico dell' istituzione, prendiamo ora in considerazione un corpus di occorrenze selezionato a partire dalle presentazioni ufficiali della nuova identità visiva ideate direttamente dell'agenzia Inarea, dalle quali tra l'altro - rifacendoci alla celebre tassonomia proposta da Floch (1995) emerge l'intenzione di adottare una strategia di comunicazione del tipo ludico-obliqua. Ecco quindi il testo principale nel quale viene proposta una estensione del lessema: "Roma come Capitale della cristianità; Roma come Capitale dello Stato italiano; Roma come Capitale della cultura; [e infine] Roma come Capitale dell'Impero romano" (16). L'identità di Roma quindi, manco a dirlo, viene valorizzata entro la dimensione della memoria del suo passato. Le tre componenti che ne riempiono la struttura riportano ai soggetti storici che l'hanno scelta come capitale, l'Impero e la chiesa romana e ultimo lo stato italiano. Soggetti difficilmente riconducibili ad un'unica identità, in quanto sono in forte contraddizione storica e politica, ma che la storia di Roma porta nel grembo delle sue complesse stratificazioni. Così il futuro della città viene fatto coincidere col suo presente storico e monumentale. Mentre non viene fatto alcun riferimento alla storia dell'istituzione. Ma ciò che più spicca in questa definizione semantica, e quindi della strategia intrapresa nell'ambito della politica dell'identità cittadina, è la posizione primaria assegnata alla componente cristiana rispetto alle altre e soprattutto a quella legata all'impero. Roma è valorizzata anzitutto per essere sorta /centralità/ del mondo religioso Cristiano.

È a partire da questa definizione programmatica, ma anche dalla rinnovata stilizzazione rinascimentale dei caratteri delle componenti verbali, che possiamo passare all'analisi delle componenti cromatiche dell'identità visiva, sempre a partire dal loro impiego nel logo di Roma capitale. Abbiamo già chiarito il significato dell'impiego del rosso quando abbiamo preso in considerazione il valore dello stemma nell'economia simbolica della cultura medioevale. Oggi certamente il rosso non ha lo stesso valore che aveva nel Medioevo, anzi serve piuttosto per richiamare alla memoria una parte della storia di Roma. Rispetto al passato tra $\mathrm{i}$ colori impiegati nelle pratiche di autorappresentazione, risulta evidente l'aggiunta del bianco che, occupando il campo del logo, copre una porzione significativa di spazio, essendo lo sfondo che ingloba e al tempo stesso emerge il soggetto che si 
rappresenta. Considerando i risultati delle analisi precedenti, la nostra ipotesi è che la riconosciuta dialettica tra cristianità e antichità propria del discorso storico e istituzionale di Roma, sia stata tradotta nel linguaggio visuale attraverso l'opposizione del bianco col rosso. Per cui siamo di fronte ad un sistema semi-simbolico che sotto intende una logica del tipo: il "bianco" sta alla cristianità rinascimentale, cosi come il "rosso" sta all'antichità classica e medioevale.

Nelle pratiche autorappresentazione considerate sinora dunque le componenti cromatiche hanno la funzione di attivare una relazione congiuntiva tra l'istituzione e il passato. Ma rispetto al passato cambiano le connotazioni: certamente ai giorni nostri nessuno interpreterebbe il rosso dello stemma come simbolo di una lotta tesa a conquistare la sovranità del popolo. E ciò perché i segni riconducibili al mito assumono un diverso significato a seconda della sfera storica e culturale nella quale vengono inseriti: se infatti nel Medioevo essi traducevano un sentimento di attesa appassionata per la restaurazione del passato, oggi diversamente gli stessi segni assumono una funzione connotativa tesa tuttalpiù a richiamare l'idea quasi turistica di una romanità antica, senza che qualcuno si aspetti di tornare a vivere realmente il mito dell'Impero.

\section{Il sistema dei logotipi capitolini}

Finora abbiamo tentato di mostrare come attraverso diversi sistemi e formazioni semiotiche il potere abbia nei secoli costruito un proprio simulacro istituzionale, ma anche come questi processi, per essere compresi, debbano essere messi in correlazione con le passioni e le coscienze collettive, politiche e civiche, della popolazione che l'istituzione vuole o deve governare. Altresì, studiando i conflitti simbolici che nel Medioevo erano tesi a ridefinire i rapporti tra le diverse forze che al tempo costituivano il sistema di potere - ovvero il Papato, il Comune e l'Impero - abbiamo visto come le strategie messe in campo fossero caratterizzate da un linguaggio comune (cfr. Sedda 2012, pp. 94-98), un codice di tipo prevalentemente simbolico e tendente ad affermare una continuità con l'antico impero romano. Vi è quindi un discorso, con una propria semantica e sintassi, della quale l'enunciatore istituzionale deve tener conto nel momento in cui costruisce il proprio simulacro. Ci riferiamo a quel "sistema di sapere", l'episteme diremo con Foucault, che da forma a ogni prassi sociale, compresa quella enunciativa.

E' stato Jean Marie Floch, già dai primi anni novanta, nel suo pionieristico e ormai classico volume sull'identità visiva (1997) a mostrare che la comunicazione ha una "cultura" propria, con modelli, credenze, sensibilità, modi di fare specifici, a partire dalla quale i pubblicitari e più in generale i "comunicatori" ideano e gestiscono, più o meno consapevolmente, il proprio lavoro. In particolare, ciò che Floch aveva studiato e mostrato, era la sempre più pressante necessità per un soggetto che viene proposto sul mercato, e che quindi è sottoposto ad un regime di consenso, di dotarsi di quella che lui stesso definì identità visiva, ovvero di tratti plastici e figurativi, da utilizzare in ogni occorrenza testuale, al fine di segnarne la cifra estetica, la coerenza e l'immediata riconoscibilità. Dotare i soggetti di un'identità visiva è 
divenuto negli anni una delle attività aziendali di maggiore importanza, e le istituzioni non sfuggono a questa tendenza.

Così, anche Roma Capitale, nella gestione della propria comunicazione istituzionale si è dotata di un sistema di identità visiva - anzi è la maggiore consapevolezza della sua forza e una sua più attenta gestione che segna una rottura rispetto al passato - del quale il logo risulta essere centro centrifugo e centripeto in uno stesso tempo, anche se non è l'unico supporto testuale sul quale essa viene proiettata. Rimanendo per il momento a questo genere, possiamo comunque notare come i logo assegnati alle varie utilites, cioè gli enti preposti alla gestione concreta dei servizi pubblici, rispettino il regime di identità visiva centrale, creando così un "attore collettivo" distinguibile come una sorta di "totalità partitiva" (Greimas, 1976). Faremo quindi ora una rapida carrellata di questa costellazione di logo e logotipi, anche mostrando come essi siano cambiati rispetto al periodo in cui l'ente si chiamava ancora Comune di Roma, per poi infine vedere come l'identità visiva venga proiettata su nuovi supporti testuali.

Ama

Il primo logo che prenderemo in considerazione sarà quello dell'AMA, la società di Roma Capitale delegata alla cura dell'ambiente e del decoro urbano.

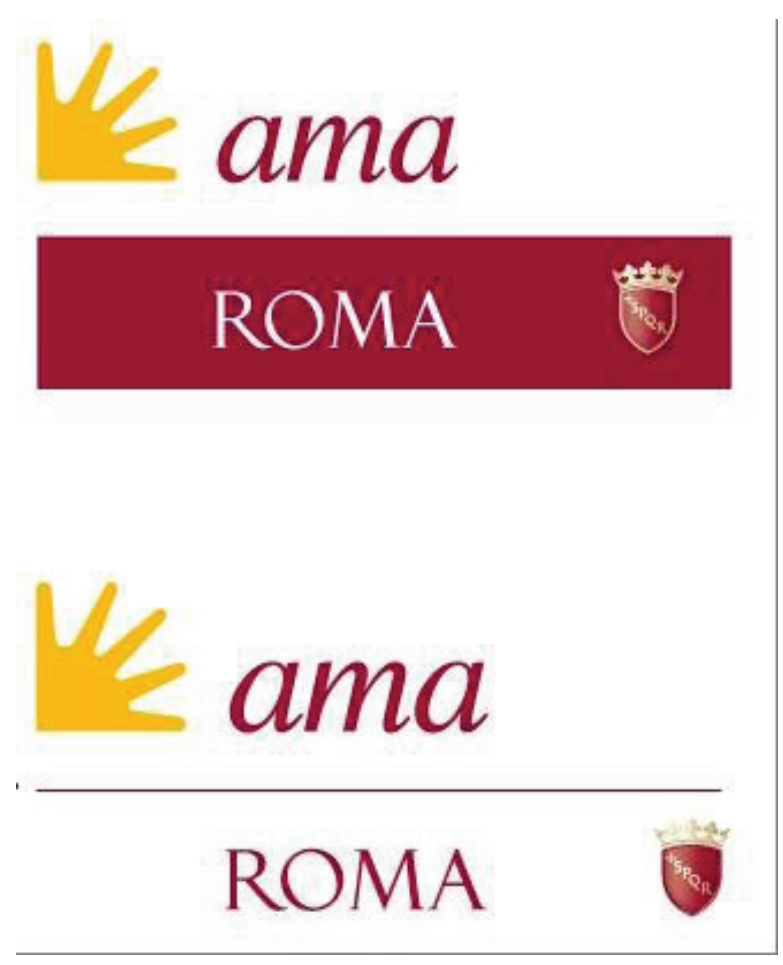

Logo AMA nelle versioni negativo e positivo.

Il logo dell'Ama, come possiamo osservare (fig. 14), si compone di due parti principali divise lungo l'asse verticale. Queste sono ulteriormente divise sul piano orizzontale in una componente figurativa e una testuale. L'evidente sistema semisimbolico mette chiaramente in relazione Ama, in alto, con l'Amministrazione 
centrale, nella parte in basso. Interessante è il complesso sistema di rinvii e rimandi attraverso cui viene creato un effetto di coerenza interna e esterna al testo, attraverso il re-impiego dei tratti plastici e figurativi dell'identità visiva di Roma Capitale. Infatti, osservando le componenti visive del logo, possiamo notare come il simbolo dell'Ama, assimilabile ad una sorta di spicchio di sole o anche a una mano aperta, sia una stilizzazione dei cinque fioroni della corona apposta sullo storico stemma del Comune; mentre rispetto al testo verbale troviamo inscritte una rima tipografi$\mathrm{ca}$ - il font usato per l'insegna Ama è il corsivo del carattere Urbs -, e una cromatica, infatti sia per la scritta superiore che per quella inferiore è stato utilizzato il rosso porpora dello stemma. Ancora, sia nella parte bassa che in quella alta, ritornano i colori rosso, giallo e bianco.

Una breve nota merita il senso veicolato dal testo che per mezzo di un semplice gioco di parole traduce il servizio di cura dell'ambiente nel tema dell'amore per città, chiedendo tra l'altro al suo enunciatore di farsene carico, se non di identificarsi con esso, come quando il logo è posto su superfici riflettenti (vedi figura 15).

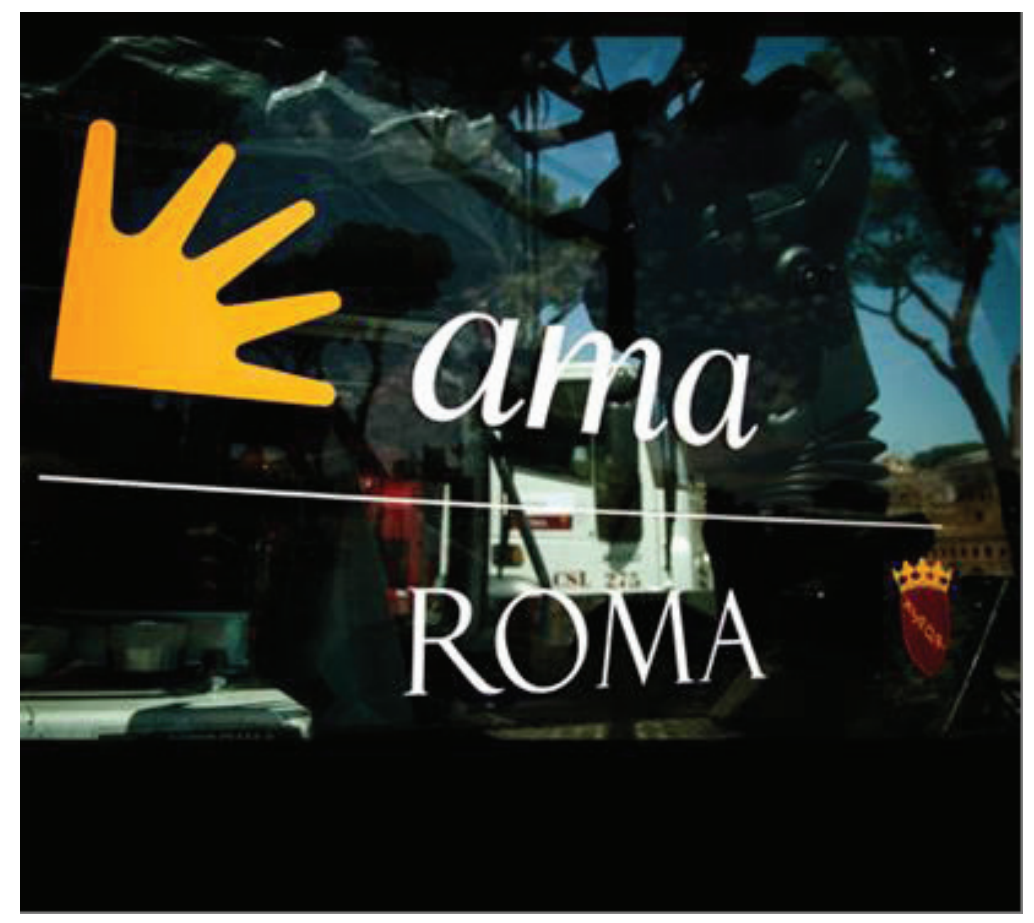

Logo AMA prima della nuova identità visiva di Roma Capitale.

Una funzione importante per i processi di significazione è poi svolta dalla categorie eidetiche, cioè dell'organizzazione e distribuzione delle linee nel testo nel nostro caso è "spigolosa" la parte bassa del testo mentre "arrotondata" quella alta - le quali, per opposizione, fanno sì che l'immagine dell'Ama risulti meno fredda e austera rispetto a quella del Comune. Questo tipo di comunicazione, che con Floch potremmo definire ludico-obliqua, l'effetto di senso che essa produce, e il tutto sommato auspicabile messaggio d'amore per la città che veicola, risultano ancora più evidenti se, così giusto per dare un'idea, la mettiamo in comparazione con quella del vecchio logo (fig. 16). 


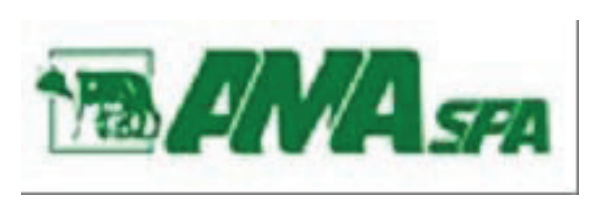

Logo AMA prima della nuova identità visiva di Roma Capitale.

Come possiamo osservare, questo richiamava una certa idea di appartenenza alla romanità classica, attraverso la figura della lupa capitolina, e dell'ambiente attraverso l'impiego monocromatico del verde. In effetti però non attivava alcuna relazione congiuntiva né tra l'azienda e l'amministrazione centrale, né tra essa e il rapporto con i cittadini.

\section{Atac, Trambus, Metro}

Atac è l'azienda per la mobilità a cui è affidata la regia delle società Trambus e Metro che erogano, rispettivamente, il servizio di trasporto pubblico di superfice e quello sotterraneo, per conto di Roma Capitale. A conferma della presenza di una forte volontà di creare una identità visiva particolarmente integrata, i logo delle tre agenzie sono in tutto identici tranne ovviamente nella parte che reca il nome dell'utility. Inoltre, anche nel caso dei logo Atac e delle due agenzie affiliate, troviamo la stessa organizzazione spaziale che caratterizza il logo dell'Ama. Questi logo sono infatti divisi in una parte alta, dove è posto il nome dell'utilty, e una bassa che, per il nome, il carattere usato, lo stemma e il colore del campo, riconduce all'identità dell'amministrazione centrale (fig. 17).

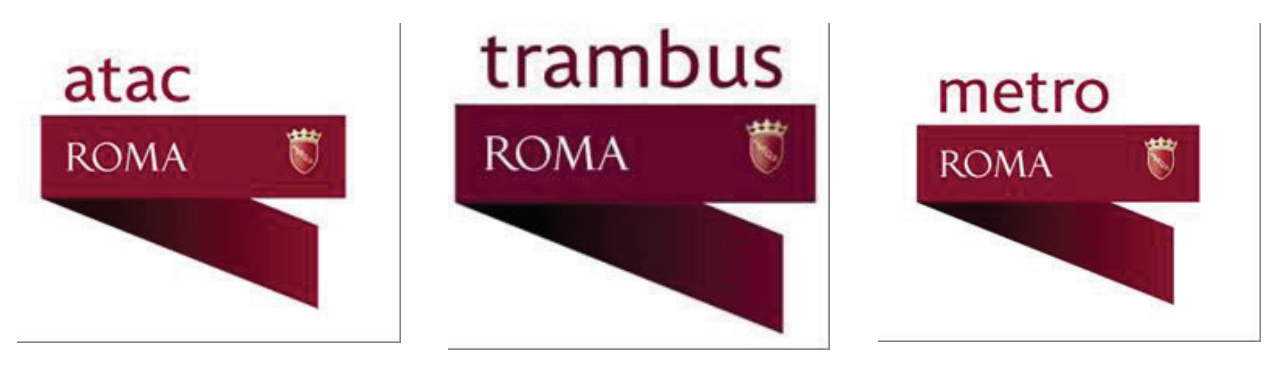

Logo Atac, Trambus, Metro.

Anche per quanto riguarda i nomi delle tre società ritroviamo il carattere l'Urbs, questa volta però nella versione “display regular” (Inarea). La comparazione con il logo dell'Ama mette in evidenza l'assenza del simbolo prima del nome della società. Questo in realtà è stato ricavato nella parte in basso, attraverso l'aggiunta alla barra rossa (che negli altri logo aveva la funzione di campo del lessema Roma e del simbolo della città) di una piega. Questa traduzione e condensazione dell'identità dell'azienda in una immagine, evoca un senso di dinamicità e, assomigliando ad una freccia, di direzionalità e rapidità, ma anche di connettività, in quanto a guardar bene la freccia può essere intesa anche come una stilizzazione dei collegamenti urbani. Il "nastro rosso" (Inarea) rappresenta quindi una sorta di "filo conduttore", che mette in congiunzione non solo le tre società ma tutti gli spazi urbani.

Anche nel caso di Atac è interessante procedere ad una comparazione dei nuovi logo con quelli vecchi. Basta riportarli con l'identica disposizione che abbiamo usato per i nuovi per rendere evidente l'attuale tensione dell'enunciatore istituzionale verso un'identità visiva 
fortemente coerente, tensione che plasma le sue pratiche di rappresentazione.

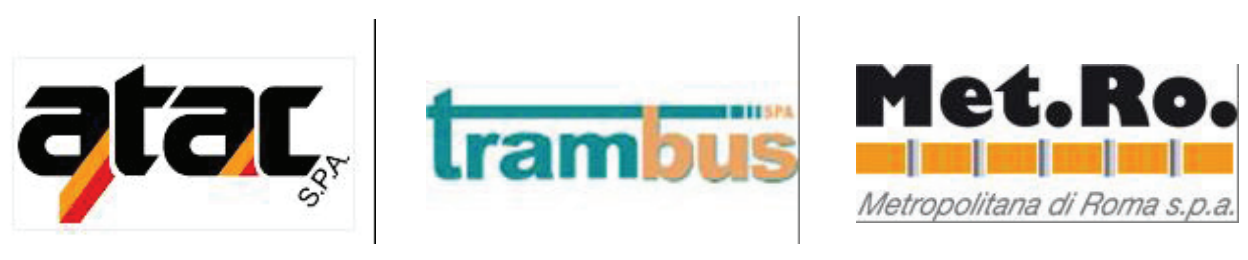

Vecchi logo dell'agenzia della mobilità.

La traduzione dell'identità visiva nei paesaggi metropolitani

Interrompiamo qui l'analisi dei logo dell'amministrazione capitolina per prendere in esame la proiezione dell'identità visiva in altri supporti testuali, e in particolari su quello che la rendono presente nello spazio pubblico. Ci riferiamo alla recente traduzione dell'identità visiva nei mezzi e nelle stazioni autobus e metropolitane. Loperazione ci sembra interessante se non altro perché è proprio in questi spazi che essa, l'identità visiva, sembra imporsi con maggiore efficacia, in quanto diviene "paesaggio", e va così a caratterizzare l'aspetto visivo della città e con esso l'esperienza del cittadino e del visitatore. Per questa analisi abbiamo selezionato un piccolo corpus di immagini rappresentanti i mezzi e le stazioni degli autobus e delle metro così come essi sono stati disegnati e realizzati nel corso del 2012 da Inarea, l'azienda di comunicazione a cui è affidata la cura dell'identità visiva della città.
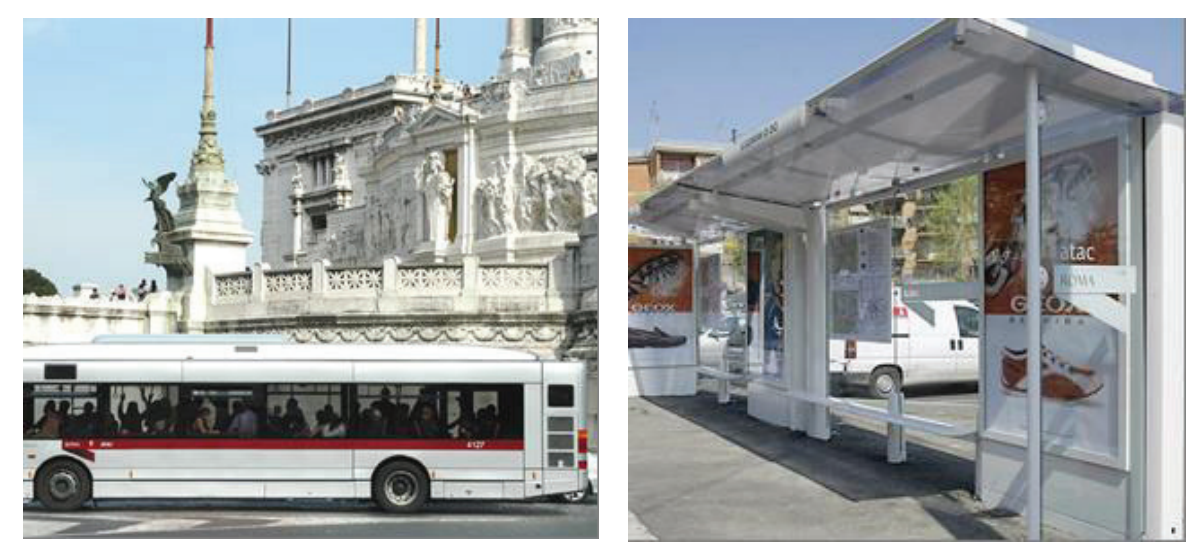

Mezzi e stazioni degli autobus

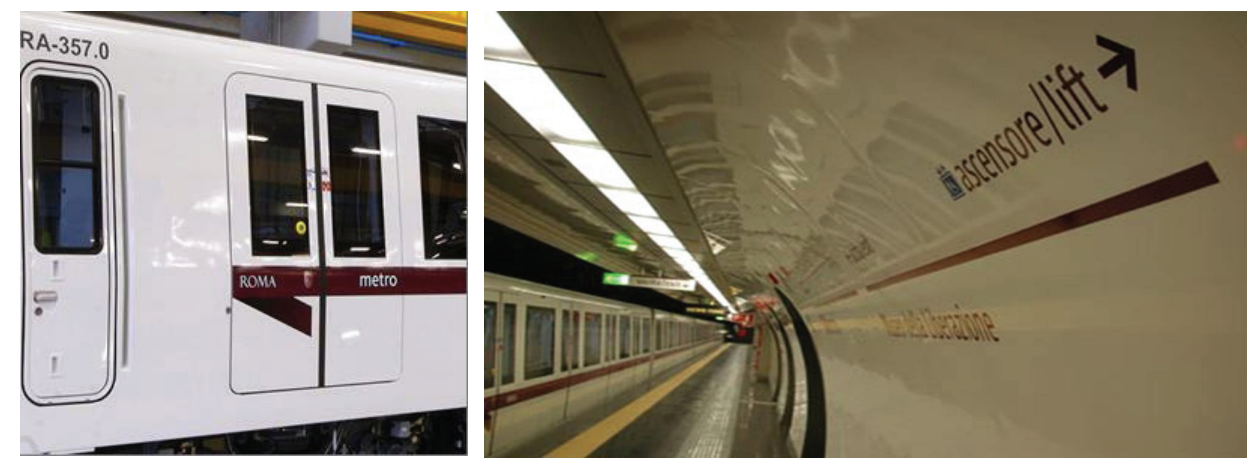

Mezzi e stazioni della metropolitana 
Lungi dal voler percorrere un'analisi esaustiva dei mezzi e delle stazioni metropolitane, tantomeno della loro fruizione da parte dell'utenza - come tra l'altro ha fatto in modo esemplare Jean Marie Floch (1990) in uno dei suoi memorabili saggi -, ai fini della nostra ricerca ci basterà mettere in relazione questo piccolo corpus con l'identità visiva, per comprendere come essa sia stata tradotta nello spazio urbano, divenendo come abbiamo detto "paesaggio". Insistiamo su questo concetto perché riteniamo che è proprio nell'operazione di proiezione dell'identità visiva nei supporti testuali metropolitani che l'enunciatore istituzionale imprime e impone le proprie marche nella città, assegnandole e definendone così l'identità. Infatti, come ha mostrato il semiologo Pierluigi Cervelli nel suo studio proprio dedicato a Roma e intitolato La città fragile (2008), è proprio l'intervento sul paesaggio urbano a costituire la modalità più performante attraverso cui l'Istituzione di potere può realizzare le proprie politiche identitarie. Un identità quella proiettata dell'Istituzione sulla città che si badi bene oltre ad essere istituzionale - e anzi, a maggior ragione, proprio in quanto "essere-istituzionale" - ha una natura politica o meglio dire, vista la sua costruzione semiotica, semiopolitica (Sedda 2012).

Rispetto alle strategie di costruzione del simulacro istituzionale della città, il corpus testuale che stiamo prendendo in considerazione è a nostro avviso equivalente a quello che abbiamo preso in carico considerando il periodo medioevale. Dunque, l'intervento su automezzi e stazioni metropolitane assolve la stessa funzione semiotica di quello che ai tempi della Renovatio si praticava nella costruzione del palazzo senatoriale, e cioè di autorappresentazione del sé.

Anche in questo caso non si può fare a meno di notare l'importanza dei cromatismi. E' infatti questa componente plastica del testo visuale a imporsi con maggiore forza ad efficacia. E quindi su questa che vogliamo soffermarci. Basta un rapido sguardo alle fotografie proposte poco più sopra (fig. 19 e 22) per rendersi conto della dominanza quasi monocromatica del colore bianco. E' quindi forse questo il colore che l'enunciatore istituzionale ha selezionato per la rappresentazione della città di Roma? E quale strategia si nasconde dietro l'impiego di questo colore-simbolo? È vero che abbiamo già parlato del valore dei colori nell'identità visiva e nel discorso istituzionale di Roma Capitale, ma qui è bene riproporre la nostra ipotesi con una sorta di ragionamento i cui argomenti avanzano nella forma di domande: se il rosso, come abbiamo mostrato, veniva utilizzato dai romani del Medioevo come colore simbolo della Repubblica, il bianco, ora impiegato in modo dominante per colorare i mezzi e le stazioni di Roma Capitale, a quale scopo viene utilizzato? Non rimanda forse anch'esso ad un periodo storico? A questa domanda possiamo rispondere con un'altra: portando il confronto su altri luoghi che contraddistinguono il paesaggio architettonico romano, se il rosso ci riporta alle facciate in mattone e alle pareti affrescate dei palazzi romani, il bianco a quali facciate ci riporta se non a quelle in travertino delle bellissime chiese barocche volete dai Papi del rinascimento? E ancora, se il rosso è il colore usato nel campo dello stemma del comune, il bianco non è forse il colore del campo della bandiera vaticana? Troppi indizi ci portano a concludere che, nell'attuale strategia di Roma capitale, dietro la 
scelta dell'impiego del bianco ci sia una volontà di rappresentazione della Roma dei papi, o meglio - visti i risultati delle analisi precedenti - di una volontà di appoggiare la costruzione dell'identità della città al mito della cristianità.

\section{La definizione della Cittadinanza nell'identità visiva}

Riprenderemo in conclusione il discorso sull'identità dalla Città. Ora invece vorremo fare una breve riflessione sugli attori dell'enunciazione presupposti nel discorso istituzionale che abbiamo sinora analizzato. È chiaro infatti che quello analizzato non è solo un discorso su Roma, ma anche un discorso che coinvolge e presuppone una definizione semantica di altri due attori: l'Istituzione e suoi Cittadini. La nuova gestione dell'identità visiva se messa in comparazione con la vecchia "costellazione di simboli" (SEDDA, 2012) di epoca medioevale, offre a riguardo un buon tramite attraverso cui cogliere sia la definizione data a questi due attori sociali, sia lo statuto del loro rapporto.

Sotto questo punto di vista è evidente che se nel passato medioevale del comune, le pratiche di rappresentazione simbolica si inscrivevano entro un' isotopia del conflitto, nella forma della lotta rivoluzionaria, all'interno della quale il Cittadino - che, lo si ricorderà, era identificato con la figura del leone - veniva caricato di valori come la "forza" e la "fedeltà" all'Istituzione, ora è altrettanto evidente che la gestione dell'identità visiva segue altre logiche, che sono proprie del discorso sulla comunicazione contemporanea. Entro questo discorso, il cittadino e l'istituzione sono le parti di un rapporto di servizio che il secondo eroga al primo. È quindi l'istituzione a dover costruire e continuamente rinsaldare quel "patto di fiducia" proprio di ogni relazione sociale. Da qui l'attenzione alla identità visive, per veicolare quei valori di "coerenza" e "trasparenza" (propri dei sistemi fortemente riconoscibili), ma anche "innovazione" (attraverso i cambiamenti delle vesti grafiche), che sono dettati dagli attuali regimi di "comunicazione istituzionale".

Bisogna però chiarire che questi valori sono affermati all'interno di un discorso "scientifico" sulla comunicazione, e in particolare sulla gestione delle identità visive, che funziona a livello "globale", ma che a nostro avviso non riflette sulle implicazioni culturali dovute alle sue ideazioni a livello "locale". Mi riferisco in particolare alla relazione che lega le identità visive con la memoria e la tradizione del luogo e dei soggetti che rappresentano, materia a partire dalla quale, come abbiamo visto e come chiariremo ulteriormente in conclusione, l'Istituzione da forma all'identità.

\section{Conclusioni. L'Istituzione tra vecchi e nuovi miti di Roma}

Per concludere tentiamo ora di tirare le fila del nostro discorso. Il nostro percorso di ricerca, che è iniziato ponendoci un interrogativo sul significato dello storico emblema del Comune di Roma, ci ha portati man mano, ma sempre in modo programmatico e cioè in linea con le recenti indagini svolte nell'ambito della semiotica della cultura (cfr. Sedda), a guidare la nostra attenzione e sensibilità, in 
una parola, il nostro sguardo, su porzioni di manifestazioni culturali via via più ampie ed eterogenee, nel tentativo di cogliere le correlazioni esistenti tra le diverse formazioni semiotiche comprese nel nostro corpus - stemmi, monete, pratiche, architetture, discorsi, etc. - le quali, se pure sono distanti nel tempo storico e nello spazio dei generi testuali, si sono rivelate molte più vicine di quanto immaginassimo, ma soprattutto euristiche e significative, rispetto al discorso storico e culturale che qui, seppure in modo sintetico, abbiamo provato a ricostruire.

Nel quadro astratto del discorso teoretico, la ricerca sullo stemma è stata anzitutto una ricerca semiotica sulle pratiche di rappresentazione simbolica tese alla costruzione dei simulacri del potere istituzionale, una ricerca durante la quale sono state individuate e - almeno nelle nostre intenzioni - mostrate le correlazioni tra queste "pratiche" e le "formazioni discorsive" che, con il Foucault di "Archeologia del Sapere" (1968), possiamo definire l'episteme della nostra cultura-oggetto. E' bene però a questo punto chiarire una posizione epistemologica importante, non vogliamo infatti far intendere che il processo di costruzione del simulacro istituzionale realizzato con le pratiche di autorappresentazione, sia per così dire "soltanto" simulacrale, ossia fuori di apparenza diversa cosa dalla "realtà", tutt’altro, come ha dimostrato Paolo Fabbri nell'importante serie di saggi raccolti nel volume "La svolta semiotica" (1998), essa, la realtà è proprio il risultato di un processo di costruzione semiotica, per questo motivo la semiotica, intesa come ricerca scientifica, deve farsene carico.

La storia del comune di Roma, la sua fondazione come Senato e quindi come restaurazione della celebre istituzione che caratterizzò la forma di governo della repubblica romana, le sue autorappresentazioni delle quali abbiamo analizzato diverse manifestazioni testuali riconducibili ad un unico discorso di potere - dalla scelta dello stemma, al luogo della sua sede istituzionale, dai discorsi politici alla sua monetazione, eccetera - mostra che la costruzione del simulacro istituzionale $\mathrm{o}$, più semplicemente, la sua identità e immagine sociale, sia stata fondata su un mito originario della città e della grandezza di Roma, ovvero quello della Repubblica che dapprima liberò i romani dalla sottomissione agli etruschi e che poi divenne Impero. Non si può capire però questa scelta strategica dei fondatori senza prendere in considerazione i rapporti di forza tra i poteri che si contendevano il dominio sulla Città, e quindi senza capire la relazione tra il comune di Roma e lo stato della Chiesa e, forse secondariamente ma comunque necessariamente, con il Sacro Romano Impero.

Abbiamo visto infatti che i romani si costituirono in Comune proprio per andare contro l'ingerenza e l'interferenza del papato nelle loro questioni economiche e nei loro affari. Fu in particolare l'intervento di Innocenzo II mirato a privare i romani dei frutti della vittoria su Tivoli, a far esplodere un processo che comunque era in atto da tempo nella gloriosa e non violenta rivoluzione del 1143. Appoggiarsi al mito della Repubblica seguiva quindi una logica di assimilazione storica: così come i romani si ribellarono agli etruschi e fondarono la Repubblica, allo stesso modo i romani del Medioevo si ribellavano al Papato per rifondare il Senato. 
D’altra parte però questa eredità, la discendenza dalla gloriosa antichità, era contesa proprio con lo stato della Chiesa il quale sicuramente le assegnava un senso meno rivoluzionario ma non meno efficace nel veicolare l'immagine di un potere che, proprio per la "grandezza" dalla quale si dichiara discendente, legittima la propria autorità e sugella così la propria credibilità agli occhi del popolo e degli altri poteri.

Per il popolo romano d'altronde quello della Repubblica era un mito con il quale, a diversi livelli di conoscenza, ci si rapportava nella quotidianità. I resti e le rovine dei palazzi e delle costruzioni antiche non erano solo componenti dei paesaggi urbani per così dire storici, ma veri e propri oggetti semiotici con i quali ri-costruire gli edifici adibiti all'uso abitativo e lavorativo. In altri termini, i romani in epoca medioevale ri-utilizzavano frammenti di antichi palazzi e rovine come un bricoleur riutilizzerebbe ingegnosamente i materiali di vecchi oggetti per costruirne di nuovi. Ma come mostrano i resti della facciata medioevale del Campidoglio e quella di Casa Crescenzi - per restare agli esempi che abbiamo fatto - questi materiali non avevano funzione meramente pratica, tutt'altro, questa era quasi "negata" al fine di affermare una valorizzazione eminentemente mitica, ovvero di costruzione e rappresentazione retorica della propria identità. Sono le testimonianze raccolte nel corpus a rivelarci che il rapporto estetico dei romani con l'antichità era dominato anzitutto da un sentimento misto di orgoglio e nostalgia, unito alla speranza e attesa di una "rinascita" del passato. È a partire da questa passione, da questa "attesa” (Pezzini 1998), che i romani basarono la propria retorica di fondazione e con essa il simulacro istituzionale, definendo in ogni modo il proprio gesto rivoluzionario una Renovatio.

Già ai tempi i poteri avevano capito che i conflitti tra di loro dovevano svolgersi entro una dimensione di persuasione della popolazione, in una serie di botta e risposta a suon di - non sanguinolente ma di certo efficaci - battaglie simboliche, mirate a legittimare agli occhi del popolo il proprio potere (SEDDA, 2012). Da qui la rincorsa e l'affrettarsi del comune per ergere subitaneamente i più svariati simboli legati all'antichità, in una "guerriglia semiologica" urbana, per usare una famosa espressione di Umberto Eco (1973), che vedeva il comune nell'intento di sottrarre porzioni di potere al Papato. È dentro questa dimensione dialogica, questo conflitto simbolico, che possiamo rendere intellegibili alcuni dei gesti (riconducibili comunque ad un'unica pratica) che abbiamo ripreso nella nostra piccola ricerca: la presa del Campidoglio, la costruzione del Palazzo Senatorio, la collocazione nella sua piazza di un obelisco sorretto da leoni, l'affissione sulla facciata del suo emblema - cioè dello scudo che è ancora tutt'oggi lo stemma della città di Roma - che con l'inscrizione dell'antico motto Senatus et PopulosQue Romanus su campo rosso pompeiano, condensava (e condensa) tanto efficacemente i valori e il ricordo dell'Impero.

Se però il mito della repubblica rappresentò il tramite a partire dal quale i romani legittimarono il proprio potere (distinguendosi dal potere della Chiesa che a sua volta e con altre ragioni basava il proprio sul mito dell'impero), assumendo così una funzione di destinante del Potere, esso non può essere considerato come 
una "modello". In altri termini, i romani non riuscirono mai a realizzarsi in un soggetto politico all'altezza del mito che avevano assunto quale proprio destinante (cf. VAUCHEZ, 2001). E ciò perché erano cambiate le condizioni sociali e culturali entro le quali il Comune agiva e soprattutto i rapporti di forza con i quali misurarsi e competere per la propria affermazione. Il papato infatti era un potere troppo grande e consolidato per poterlo scavalcare con facilità. Lo dimostra il mancato riconoscimento del Senato da parte del Sacro Romano Impero, che rifiutò di dar seguito alle richieste dei romani quando, e in tal senso è emblematico il discorso di Cola di Rienzo, questi chiesero il rispetto della lex regia, rivendicando con essa il diritto di proclamare l'imperatore, diritto che con la fine dell'impero romano era passato dalle mani del senato a quelle del papa.

È proprio con la morte di Cola di Rienzo, celebrato da Petrarca come colui che replicò il gesto rivoluzionario dei romani della repubblica, che si concluse l'esperienza di affrancamento della storia di Roma dal potere del papato. Lo stato della Chiesa da lì in poi dominò la città cambiandone completamente l'assetto urbano e architettonico e al tempo stesso determinando nel tempo un nuovo mito della città, il mito della Roma cristiana.

$\grave{E}$ a partire dalla fine del comune libero che la nostra ricerca ha dovuto fare un salto lungo cinque secoli per riprendere il suo cammino dalla ritrovata autonomia del comune dai poteri del Vaticano e con essa dalla riavvio delle pratiche di autorappresentazione e quindi di costruzione del proprio simulacro istituzionale. In particolare ci siamo soffermati sugli effetti dell'ultima riforma istituzionale che ha mutato lo statuto giuridico dell'amministrazione. Una riforma importante, che ha cambiato l'assetto amministrativo dell' istituzione sin dal suo nome, questa infatti non si chiama più "comune di Roma" ma "Roma Capitale". Non ci interessa qui riprendere tutto il percorso di ricerca ma solo portare alle estreme conseguenze le possibilità offerte da una comparazione effettuata sull'asse diacronico, sottolineando le differenze e i cambiamenti sul piano delle strategie di posizionamento, di autodefinizione del sé istituzionale.

A questo riguardo, tenendo il punto sulle pratiche di autorappresentazione, la prima evidenza riguarda il cambiamento rispetto ai modi in cui esse si danno: sono cambiati cioè i generi testuali nei quali vengono costruite e affermate le identità sociali (nel nostro caso potremmo parlare di enunciati istituzionali) attraverso la loro traduzione (sempre "imperfetta" come ha dimostrato Franciscu Sedda nel suo sistematico studio sulle traduzioni culturali) in differenti piani dell'espressione. Se prima l'identità del comune veniva veicolata attraverso pratiche che la condensavano nello stemma o in altri generi testuali (come quello architettonico, solo per riprendere l'esempio del Campidoglio), ora la costruzione dell'identità segue più complesse codificazioni, create nell'ambito di un "discorso scientifico sulla comunicazione" che ha trasformato le pratiche autorappresentazione in rigorosi protocolli (FONTANILLE, 2010) di gestione delle "identità visive". Ovvero si parte da dei tratti plastici e figurativi dell'immagine per poi tradurli in differenti generi testuali e forme di comunicazione legate all'Ente pubblico: logotipi, documenti, 
manifesti, siti web, etc.

Nel nostro caso, abbiamo analizzato le pratiche di rappresentazione dell'identità visiva di Roma Capitale, a partire dal sistema dei logotipi che ha assegnato all'ente centrale e alle sue due utility principali, quelle che si occupano dell'erogazione del servizio trasporti e della cura dell'ambiente. E' risultata così evidente l'importanza delle componenti cromatiche nel sistema dell'identità visiva: il nuovo logo presenta un vasto campo bianco sul quale sono inscritti, in rosso, lo storico stemma e il nuovo nome dell'istituzione, Roma Capitale.

Per rendere però intellegibile l'identità visiva e quindi per tradurne i contenuti, è stato necessario ancora una volta mettere in comparazione le sue manifestazioni testuali con altri testi per così dire "programmatici" dello stesso ente. È in particolare nello statuto del comune, laddove si vuole dare una estensione al nome Roma Capitale per chiarire cosa esso voglia significare, che si legge, in ordine, che Roma è "capitale della Cristianità, dello Stato italiano, e del patrimonio storico e culturale".

Senza ripetere qui l'analisi, e facendo una rapida comparazione con la costellazione di simboli che esprimeva l'identità del comune medioevale, è chiaro quindi che così come il "rosso" serviva nel Medioevo per evocare il mito della Repubblica, così ora il "bianco" serve per evocare quello della Cristianità.

Rispetto alle implicazioni di questa neonata identità cittadina, teniamo a ribadire che la convocazione nel discorso istituzionale dell'uno o dell'altro periodo della storia della città attraverso l'uso simbolico del colore, significa attivare nella memoria un diverso senso della romanità, la quale può essere dipinta per cosi dire "laicamente" attraverso il richiamo al mito dell'impero e al corollario di sentimenti e valori di orgoglio, audacia e lotta che questa tradizione porta con se, oppure "religiosamente" facendo appello al mito della cristianità romana.

Considerando la storia dei rapporti di forza tra il papato e i romani del periodo medioevale, sembrerebbe oggi paradossale che l'attuale costruzione del simulacro istituzionale di Roma Capitale e con esso dell'immagine della Città, si appoggi proprio sul mito della Roma cristiana fondato dal papato lungo i cinque secoli del suo dominio sulla città. Ma sarebbe sbagliato inscrivere la strategia seguita da Roma Capitale entro il vecchio conflitto con il Vaticano. Il comune di Roma non deve più lottare contro il papato. Il conflitto si è spostato nella dimensione dell'attuale economia di mercato del mondo globalizzato che vede le grandi metropoli competere tra loro per far confluire in esse capitali finanziari ed economici. Tra questi ci sono quelli indotti dal mercato del turismo e basta guardare i dati sui turisti che ogni anno vengono a Roma attratti dalla sua identità cristiana per trovare un senso al rinnovato mito di Roma, che non sia legato solo al rapporto di questa città col suo passato, rapporto che sembra essere castrante di ogni potenziale slancio verso il futuro.

Con ciò non intendiamo certo prendere posizione o dare un giudizio di valore sulle scelte strategiche relative alle politiche dell'identità di Roma capitale. 
Tantomeno vogliamo far passare il nostro lavoro per definitivo. Le identità come si è visto sono oggetto di continui processi di negoziazione intersoggettiva che ne producono mutamenti sia sul piano dell'espressione, sia su quello del contenuto. Prova ne sia, se non bastasse il percorso compiuto sinora, che proprio nei giorni in cui concludiamo il nostro articolo, sta prendendo piede nella città l'ultimo intervento politico del sindaco Gianni Almenno che, prima di cedere il governo della città alla giunta del nuovo sindaco Ignazio Marino con il quale la sinistra è tornata al comando, ha dato il via ad una ridefinizione della veste cromatica degli autobus romani, riportando alla città di Roma il suo colore più antico, e così riattivando una relazione giuntiva tra la romanità e il mito dell'Impero romano.

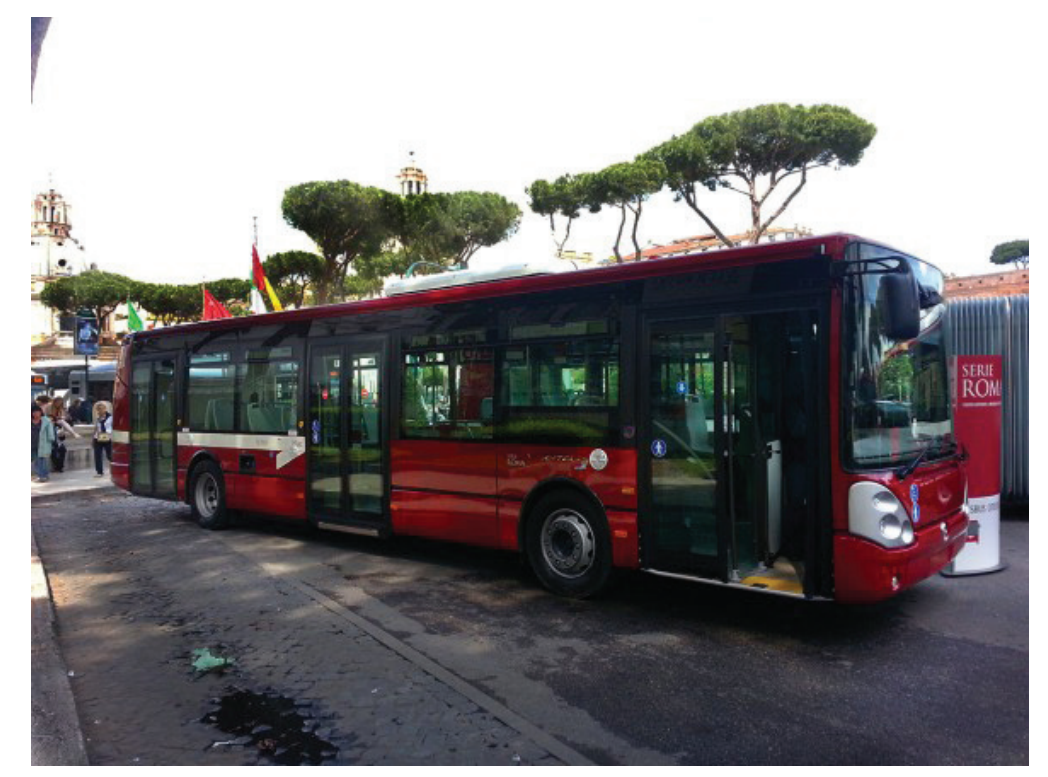




\section{Referências Bibliográficas}

APPADURAI, A. Modernity at large: cultural dimensions of globalization, Minneapolis-London, University of Minnesota Press, 1996; trad. It. Modernità in polvere. Dimensioni culturali della globalizzazione. Milano: Raffaello Cortina Editore, 2012.

CAROCCI, S. Baroni di Roma. Dominazioni signorili e lignaggi aristocratici nel duecento e nel primo Trecento. Roma: Istituto storico italiano per il Medio Evo, 1993.

CERVELLI, P., La città fragile. Roma: Lithos, 2008.

De CERTEAU, M. L'invention du quotidien. Arts du faire. Paris : Gallimard, 1990; trad. It. L'invenzione del quotidiano. Arti del fare. Roma: Edizioni Lavoro, 2001.

DELEUZE, G. Foucault. Paris: Les Editions de Minuit, 1986.

ECO, U. Il costume di casa. Milano: Bompiani, 1973.

FABBRI, P. La svolta semiotica. Bari-Roma: Laterza, 1998.

FLOCH, J. M. Semiotica, marketing e comunicazione. Dietro i segni le strategie. Milano: Franco Angeli, 1990. . Identità Visive. Milano: Franco Angeli, 1997.

FONTANILLE, J. Pratiques Sémiotiques. Paris: PUF, 2008; trad. it. Pratiche semiotiche. Pisa: Edizioni ETS, 2010.

FOUCAULT, M. Archéologie du savoir. Paris: Gallimard, 1969; trad. it. Archeologia del sapere. Milano: BUR, 1999.

Gatto, L. Storia di Roma nel Medioevo. Roma: Newton \& Compoton Editori, 1999.

GENINASCA, J. La parole littérarire. Paris: Presses Universitaires de France, 1997; trad. it. La parola letteraria. Milano: Bompiani, 2000.

GREIMAS, A. J. Sémiotique et sciences sociales, Paris, Seuil, 1976; trad. it. Semiotica e scienze sociali, Torino: Centro Scientifico Editore, 1995.

- Courtès, J. Sémiotique. Dictionaire raisonné de la théorie du langage. Paris : Seuil, 1979; trad. it. Paolo Fabbri (a cura di). Semiotica: dizionario ragionato della teoria del linguaggio. Milano: Mondadori, 2007.

GREGOROVIUS, F. Storia della città di Roma nel Medioevo. Città di Castello: Unione Arti Grafiche, (ed. or. 1859-1872) 1938-1943.

LOTMAN, J. M. La semiosfera. Lasimmetria e il dialogo nelle strutture pensanti. Venezia: Marsilio, 1985.

Marsilio, 1994.

.Cercare la strada. Modelli della cultura. Venezia: - (a cura di Sedda F.), Tesi per una semiotica delle 
culture. Roma: Meltemi, 2006.

MARRONE, G., Pezzini, I., (a cura di), Linguaggi della città, Roma, Meltemi, 2008.

. Cultura/natura, città/campagna: il caso GNAC, pp. 31-39, in Linguaggi della città. Marrone, G., Pezzini, I., (a cura di). Roma: Meltemi, 2008.

MIGLIO, M. Tradizioni popolari e coscienza politica, in Roma Medioevale, AAVV, André Vauchez (a cura di). Milano: Laterza, 2001.

PETRARCA, F. (a cura di Rossi V.) Le Familiari. Edizione Critica. Firenze: Le Lettere (Ediz Nazionale delle opere di Francesco Petrarca), 1197, 1380.

PEZZINI, I. Le passioni del lettore. Pisa: Bompiani, 1998.

Laterza, 2008.

Immagini quotidiane. Sociosemiotica visuale. Roma-Bari:

PIETRANGELI, C. Lo stemma del Comune di Roma. Capitolium, Roma, Comune di Roma, numero 2, 1953 (XXVIII), pp. 57-62, 1953 (a).

. Insegne e stemmi dei rioni di Roma. Capitolium,

Roma, Comune di Roma, numero 6, anno 1953 (XXVIII), pp. 182192, 1953 (b).

Lo stemma di Roma. Capitolium, Roma, Comune di Roma, numero 1, 1957 (XXXII), p. 9, 1957.

THESEIDER Duprè, E. Roma dal Comune di Popolo alla signoria pontificia (1252-1377), Bologna: Capelli, 1952.

RIBEZZI, A. Da Comune a Capitale. Identità visiva di Roma. Roma, Tesi di laurea in Scienze della Comunicazione, Università Sapienza di Roma, 2013.

SEDDA, F. Imperfette traduzioni. Roma: Edizioni Nuova Cultura, 2012.

VAUCHEZ, A. Introduzione, in Vauchez A. (a cura di), Roma Mediovale. Milano: Laterza, 2001.

VIGUEUR MAIRE, J.-C. Lautre Rome. Une histoire des Romains à l'époque des communes (XII-XIV siècle). Paris: Editions Tallandier, 2010; trad. it. di Paolo Garbini, L'altra Roma. Una storia dei romani all'epoca dei comuni (secoli XII-XIV), Torino: Einaudi, 2011.

\section{SITOGRAFIA}

http://www.archiviocapitolinorisorsedigitali.it

http://www.ilfattoquotidiano.it

http://www.roma-capitale.it 
http://www.inarea.com

\section{Notas}

(1) Gli articolo di Carlo Pietrangeli sono consultabili on-line sul catalogo degli archivi capitolini:

- 1953 (a) "Lo stemma del Comune di Roma" in Capitolium, numero 2, 1953 (XXVIII), pp. 57-62; http://www.archiviocapitolinorisorsedigitali.it/view_doc_frameset.php?IDA=698IDF=1965

- 1953 (b) "Insegne e stemmi dei rioni di Roma", in Capitolium, numero 6, anno 1953 (XXVIII), pp. 182-192;

http://www.archiviocapitolinorisorsedigitali.it/view_doc_frameset. php? IDA=69\&IDF $=1987$

- 1957 “Lo stemma di Roma”, in Capitolium, numero1, 1957 (XXXII), p. 9; http://www. archiviocapitolinorisorsedigitali.it/files/archivio_69/32_1957_01_15-15_00001.pdf

(2)Sulle operazione che hanno interessato l'architettura e la struttura urbanistica della città cambiandone radicalmente le forme per adeguarle agli interessi dei poteri che si sono avvicendati nella storia di Roma, vedere lo studio di Pierluigi Cervelli, "La città fragile".

(3) In tal senso si veda pure "Gregorovius F., Storia della città di Roma nel Medioevo, ed. or. 1859-1872, Unione arti grafiche, Città di Castello 1938-1943”. Entrambe le opere contengono un vizio nelle fonti in quanto, conformemente alla tradizione storiografica dell'epoca, non vi si riscontra un interesse per le dimensioni economiche e sociali della storia.

(4)Rimase solo una copia degli statuti del 1340.

(5) L'esaltazione del modello repubblicano rispetto a quello dell'impero si trova presso Petrarca. Lo sguardo dell'autore sull'impero è stato formato dalla lettura delle opere di Tito Livio, il quale ammira dei romani soprattutto le doti morali, come la pietà, il coraggio, la devozione alla patria, dimostrate durante la lotta alla monarchia e poi con l'espansione dell'impero al mondo civilizzato. Petrarca vede in Cola di Rienzo le stesse doti di Bruto: così come questo ha liberato Roma dall'ultimo re etrusco, così Cola l'ha liberata dalla tirannia dei baroni.

(6) Pietrangeli, C., Il palazzo senatorio nel Medioevo, pp.13-19, in Capitolium, 1960 , n. 35.

(7) Questo tipo di stemma "a mosaico", insieme ad un altro di forma identica, è stato scoperto nel 1889 sulla facciata del Palazzo Senatorio. Lo stemma è ora conservato nell'aula consiliare del Campidoglio.

(8) Per la sua stessa funzione come anche per il costo e le caratteristiche preziose dei suoi materiali, il mosaico era considerato un'arte superiore alla pittura. Ciò spiega 
anche la frequenza con cui il mosaico è presente sule facciate delle chiese. Obbligato a lavorare con cubetti di pietra, il mosaicista è indotto a semplificare il disegno di tutto ciò che vuole raffigurare. Ma questa semplificazione dei segni, alla quale l'artista è costretto dalla natura stessa della sua arte, può diventare una formidabile risorsa nel momento in cui viene utilizzata per dare più forza e più chiarezza al messaggio affidato all'opera. Tanto che nelle mani degli ecclesiastici, l'arte del mosaico diventa un'arte rigidamente codificata e specializzata nella trasmissione di una categoria ben precisa di messaggi (cfr. Vigueur, p. 346).

(9) Si tratta del cippo marmoreo sul quale posava l'urna cineraria di Agrippina, moglie di Germanico, proveniente dal mausoleo di Augusto e che nel XIV secolo fu convertito in unità di misura per il grano assumendo il nome di Rugitella del grano

(10) La dea, come il leone, verranno utilizzati all'interno di altri discorsi e dispositivi testuali. La figura del leone, per fare un esempio tra i tanti, la si ritrova nei sepolcri dei cittadini come segno equivalente all'espressione "romanus". Mentre, tra le altre note raffigurazioni della dea ricordiamo la presenza di un rilievo collocato nell'Ara Pacis (I sec.). Ma la dea-a testimonianza di un continuo dialogo tra le culture e le relative epoche storiche- viene ripresa anche in epoca moderna. Tra le più note: la statua presente in Piazza del Popolo (XIX secolo) e la "Dea Roma", opera di Igor Mitoraj (2003) collocata alla fine di Ponte Risorgimento partendo da Piazzale delle Belle Arti. 